\title{
A Displacement Approach to Decoding Algebraic Codes
}

\author{
V. Olshevsky and M. Amin Shokrollahi
}

\begin{abstract}
Algebraic coding theory is one of the areas that routinely gives rise to computational problems involving various structured matrices, such as Hankel, Vandermonde, Cauchy matrices, and certain generalizations thereof. Their structure has often been used to derive efficient algorithms; however, the use of the structure was pattern-specific, without applying a unified technique. In contrast, in several other areas, where structured matrices are also widely encountered, the concept of displacement rank was found to be useful to derive efficient algorithms in a unified manner (i.e., not depending on a particular pattern of structure). The latter technique allows one to "compress," in a unified way, different types of $n \times n$ structured matrices to only $\alpha n$ parameters. This typically leads to computational savings (in many applications the number $\alpha$, called the displacement rank, is a small fixed constant).

In this paper we demonstrate the power of the displacement structure approach by deriving in a unified way efficient algorithms for a number of decoding problems. We accelerate the Sudan's list decoding algorithm for ReedSolomon codes, its generalization to algebraic-geometric codes by Shokrollahi and Wasserman, and the improvement of Guruswami and Sudan in the case of Reed-Solomon codes. In particular, we notice that matrices that occur in the context of list decoding have low displacement rank, and use this fact to derive algorithms that use $O\left(n^{2} \ell\right)$ and $O\left(n^{7 / 3} \ell\right)$ operations over the base field for list decoding of Reed-Solomon codes and algebraic-geometric codes from certain plane curves, respectively. Here $\ell$ denotes the length of the list; assuming that $\ell$ is constant, this gives algorithms of running time $O\left(n^{2}\right)$ and $O\left(n^{7 / 3}\right)$, which is the same as the running time of conventional decoding algorithms. We also present efficient parallel algorithms for the above tasks.

To the best of our knowledge this is the first application of the concept of displacement rank to the unified derivation of several decoding algorithms; the technique can be useful in finding efficient and fast methods for solving other decoding problems.
\end{abstract}

\section{Introduction}

Matrices with different patterns of structure are frequently encountered in the context of coding theory. For example, Hankel matrices $H=\left[h_{i-j}\right]$ arise in the Berlekamp-Massey algorithm [3], Vandermonde matrices $V=\left[x_{i}^{j}\right]$ are encountered

1991 Mathematics Subject Classification. Primary: 11T71, 94B99, Secondary: 15A57.

Key words and phrases. Reed-Solomon codes, algebraic codes, displacement structure.

This work was supported in part by NSF contracts CCR 0098222 and 0242518. 
in the context of Reed-Solomon codes, and Cauchy matrices $C=\left[\frac{1}{x_{i}-y_{j}}\right]$ are related to the classical Goppa codes [32]. There are quite a few other examples involving certain generalizations of such matrices, e.g., paired Vandermonde matrices $V=[V \mid D V]$ with diagonal $D$ and Vandermonde $V$ occur in the context of the Welch-Berlekamp algorithm [4]. In most cases one is interested in finding a nonzero element in the kernel of the matrix. This problem has been solved for each of the above cases resulting in existing efficient algorithms. Although it is obvious that these algorithms make (often implicit) use of the structure of the underlying matrices, in each case this exploitation was limited to a particular pattern of structure.

Structured matrices appear in many other areas as well, and in some of these areas there are unified methods to derive efficient formulas and algorithms for them. Since we focus here on a particular application, it is virtually impossible to give an adequate introduction to all existing approaches here. We refer an interested reader to $[\mathbf{3 6}]$ for a survey of a rational matrix interpolation approach, to $[\mathbf{8}]$ for the description of the use of the concept of the reproducing kernels. Their techniques can also be used to derive efficient decoding algorithms. In this paper we limit ourselves to another well-known approach based on the concept of displacement structure (we provide some historical notes below). The latter method may have certain advantages over the others since it can be described using transparent linear algebra terms as follows. Let two auxiliary simple (e.g., Jordan form) matrices $F$ and $A$ be given and fixed, and let $R$ belongs to the class of matrices with the low displacement rank, the latter is defined as

$$
\alpha=\operatorname{rank}(F R-R A) .
$$

Then one can factor (non-uniquely)

$$
F R-R A=G B^{T}, \quad\left(G, B \in \mathbb{C}^{n \times \alpha}\right),
$$

i.e., both matrices on the right-hand side of (1) have only $\alpha$ columns each. Thus, the displacement rank $\alpha$ measures the complexity of $R$, because if (1) is solvable for $R$, then all its $n^{2}$ entries are described by only $2 \alpha n$ entries of $\{G, B\}$ (in most of applications the auxiliary matrices $F$ and $A$ are fixed and simple). The displacement structure approach is in solving matrix problems for $R$ by ignoring its entries and using instead only $2 \alpha n$ entries of $\{G, B\}$. Operating on $\alpha n$ parameters rather than on $n^{2}$ entries allows one to achieve computational savings. There are various types of efficient algorithms (such as inversion, Levinson-type, Schur-type, mixed, etc.) that can be derived via this approach. Until now we did not address specific patterns of structure. Several easily verified inequalities shown in Table 1 explain why the approach has been found to be useful to study Hankel, Vandermonde or Cauchy matrices. For example, for Cauchy matrices $R=\left[\frac{1}{x_{i}-y_{j}}\right]$ we have: $\operatorname{rank}\left(D_{x} R-\right.$ $\left.R D_{y}\right)=\operatorname{rank}\left[\frac{x_{i}-y_{j}}{x_{i}-y_{j}}\right]=\operatorname{rank}[1]=1$. We see that each pattern of structure in Table 1 is associated with its own choice of the auxiliary matrices $\{F, A$,$\} in (1); the name$ "displacement structure" was introduced since the approach was originally used only for Toeplitz matrices for which the auxiliary matrices are displacement (or shift) matrices $Z$. In particular, in the very first publications on this subject it was noticed that in many instances there is no difference between the classical Toeplitz matrices for which the displacement rank is 2 and the more general Toeplitz-like matrices. The latter are defined as those with a small (though possibly bigger than 


\begin{tabular}{|l|ll|}
\hline Toeplitz $R=\left[t_{i-j}\right]$ & $\alpha=\operatorname{rank}(Z R-R Z)$ & $\leq 2$ \\
Hankel $R=\left[h_{i+j}\right]$ & $\alpha=\operatorname{rank}\left(Z R-R Z^{T}\right)$ & $\leq 2$ \\
Vandermonde $R=\left[x_{i}^{j}\right]$ & $\alpha=\operatorname{rank}\left(D_{x}^{-1} R-R Z^{T}\right)=1$ \\
Cauchy $R=\left[\frac{1}{x_{i}-y_{j}}\right]$ & $\alpha=\operatorname{rank}\left(D_{x} R-R D_{y}\right)$ & $=1$ \\
\hline
\end{tabular}

TABLE 1. Here $Z^{T}=J(0)$ is one Jordan block with the eigenvalue 0 , and $\left\{D_{x}, D_{y}\right\}$ are diagonal matrices.

2) displacement rank with respect to the shift (or displacement) auxiliary matrices $F=A=Z$. We next give some references.

Perhaps the first reference explicitly discussing efficient algorithms for matrices with displacement structure is the thesis [33] where in Sec. 4.0.1 M.Morf writes: "In October 1970 the crucial shift-low-rank updating property was recognized by the author as the proper generalization of the Toeplitz and Hankel structure of matrices... The algorithm was called "Fast Cholesky decomposition" (or RMH4, the forth in a series of related algorithms, see Sec 6.1). In June 1971 computer programs for both scalar and block decompositions were successfully completed by the author. It was found that the known numerical well behavior of the Cholesky decomposition seemed to be inherited." Thesis [33] contains a plethora of ideas and interesting references; however it is not widely accessible, and unfortunately it is sometimes improperly referenced in recent reviews. The birth certificate to the concept of displacement structure was given in journal publications $[\mathbf{9}]$ and $[\mathbf{2 3}]$. However, let us also mention the paper [42] (see also [43]), where displacement equations appeared in the context of factorization of rational matrix functions, and where the crucial property of the Schur complements of matrices with displacement structure was first noticed; matrix interpretations of some Sakhnovich's results were independently derived in [34]. For completeness let us also mention a few papers dealing with continuous analogs of the concept of displacement. In $[\mathbf{4 0 , 4 1 ]}$ the idea of displacement structure in principle appears for integral operators with a difference kernel. In [21] this idea appeared in connection with the Chandrasekhar equations. We finally note that the displacement structure considered in [33] and [9] was limited to Toeplitz-like matrices. A general displacement approach was proposed in [16] and was applied in [17] to study matrices related not only to Toeplitz but also to Vandermonde and Cauchy matrices. For more information and plethora of different results and approaches we refer also to $[\mathbf{1 7}],[\mathbf{2 7}],[\mathbf{8}],[\mathbf{3 6}]$ and $[\mathbf{3 5}]$ and (complementing each other) references therein.

Though the displacement approach to developing fast algorithms for structured matrices is well-understood, it has never been applied to unify the derivation of efficient decoding algorithms. In this paper we show how to use it to derive in a unified manner a number of efficient decoding algorithms. We first derive solutions to list-decoding problems concerning Reed-Solomon- and algebraic-geometric codes. Given a received word and an integer $e$, a list decoding algorithm returns a list of all codewords which have distance at most $e$ from the received word. Building on a sequence of previous results $[\mathbf{4 6}, \mathbf{5}, \mathbf{1}]$, Sudan $[\mathbf{4 5}]$ was the first to invent an efficient list-decoding algorithm for Reed-Solomon-codes. This algorithm, its subsequent generalizations by Shokrollahi and Wasserman [44] to algebraic-geometric codes, and the recent extension by Guruswami and Sudan [15] are among the best decoding 
algorithms known in terms of the number of errors they can correct. All these algorithms run in two steps. The first step, which we call the linear algebra step, consists of computing a nonzero element in the kernel of a certain structured matrix. This element is then interpreted as a polynomial over an appropriate field; the second step, called the root-finding step tries to find the roots of this polynomial over that field. The latter is a subject of investigation of its own and can be solved very efficiently in many cases $[\mathbf{2}, \mathbf{1 1}, \mathbf{3 9}]$, so we will concentrate in this paper on the first step only. This will be done by applying our general algorithm in Sections 4 and 5. Specifically, we will for instance easily prove that the linear algebra step of decoding Reed-Solomon-codes of block length $n$ with lists of length $\ell$ can be accomplished in time $O\left(n^{2} \ell\right)$. A similar time bound holds also for the root-finding step, and so the overall running time of the list-decoding procedure is of this order of magnitude. This result matches that of Roth and Ruckenstein [39], though the latter has been obtained using completely different methods. Furthermore, we will design a novel $O\left(n^{7 / 3} \ell\right)$ algorithm for the linear algebra step of list decoding of certain algebraic-geometric-codes from plane curves of block length $n$ with lists of length $\ell$. We remark that, using other means, Høholdt and Refslund Nielsen [18] have obtained an algorithm for list decoding on Hermitian curves which solves both steps of the algorithm in [15] more efficiently.

In comparison to the existing decoding algorithms, the displacement structure approach seems to have the advantage of leading to a transparent algorithm design. For instance, it allowed us to design parallel decoding algorithms by using the same principles as those of sequential algorithms, though the details are more complicated since one needs have to modify the corresponding types of structure.

The paper is organized as follows. Sections 2 and 3 recall basic definitions of the displacement structure theory, and specify several particular versions of matrix algorithms to be used and analyzed in the rest of the paper. Sections 4, 5, and 6 apply these results to speed up Sudan's algorithm, its generalization by ShokrollahiWasserman, and the improvement of Guruswami-Sudan, respectively. The running times for these algorithms range from $O\left(\ell n^{2}\right)$ for Reed-Solomon codes to $O\left(n^{7 / 3} \ell\right)$ for AG-codes on curves from plane algebraic curves, where $n$ is the block-length of the code and $\ell$ is the list-size. Section 7 introduces methods for the construction of efficient parallel algorithms for the above tasks.

\section{The Displacement Structure Approach}

The problem of computing a nonzero element $x$ in the kernel $V x=0$ of a certain structured matrix $V$ frequently occurs in the context of constructing decoding algorithms. This is a trivial linear algebra problem; however exploiting the structure of the underlying matrix typically allows one to achieve computational savings. In this paper we solve such problem for the three kinds structured matrices (10), (13), (14) shown in the main text below. Before addressing particular details for each of these matrices in sec. 4,5 and 6 , resp. we specify in this section section some general frameworks for finding kernel vectors for these matrices, the latter task can be solved by using Gaussian elimination for computing a $P L U$-decomposition $V=P L U$ with a permutation matrix $P$, an invertible lower triangular matrix $L$, and an upper triangular matrix $U$. We would like to give a succinct presentation of this well-known procedure, as it is necessary to set up the notations and it is useful for the understanding of the algorithms presented below. 
It is well-known (cf. with [10]) that applying Gaussian elimination to an arbitrary matrix $V$ is equivalent to recursive Schur complementation, as shown by factorization

$$
V=\left(\begin{array}{cc}
v & V_{12} \\
V_{21} & V_{22}
\end{array}\right)=\left(\begin{array}{cc}
1 & 0 \\
V_{21} \frac{1}{v} & I
\end{array}\right) \cdot\left(\begin{array}{cc}
v & V_{12} \\
0 & V_{2}
\end{array}\right)
$$

where $V_{2}=V_{22}-V_{21} \frac{1}{v} V_{12}$ is the Schur complement of the $(1,1)$ entry $v$ in the matrix $V$. Applying (2) recursively to compute $V_{2}=L_{2} U_{2}$ one obtains the $L U$ factorization for the entire matrix $V$ :

$$
V=\underbrace{\left(\begin{array}{cc}
1 & 0 \\
V_{21} / v & L_{2}
\end{array}\right)}_{=: L} \cdot \underbrace{\left(\begin{array}{cc}
v & V_{21} \\
0 & U_{2}
\end{array}\right)}_{=: U} .
$$

This is the basis for the Gaussian elimination algorithm, which iteratively computes Schur-complements and an $L U$-decomposition thereof. Throughout the above discussion we assumed $v \neq 0$ which is not always true. This can be easily resolved by pivoting (row interchanges), so that the algorithm additionally produces a permutation matrix $Q$ such that $Q V$ has the property that the $(1,1)$-entry of all the Schur-complements are nonzero, and hence the above procedure is applicable. Altogether, the algorithm produces a decomposition $V=P L U$, where $P=Q^{-1}$.

Note that it is trivial to compute a nonzero element in the kernel of $V$ once a $P L U$-decomposition is known, since the kernel of $V$ and that of $U$ coincide, and a nonzero element in the latter can be found using backward substitution.

It is now well known that the above procedure can be speeded up in the case matrix $V$ has a displacement structure as in

$$
\left(\begin{array}{cc}
d_{1} & \mathbf{0} \\
\star & D_{2}
\end{array}\right) V-V\left(\begin{array}{cc}
a_{1} & \star \\
\mathbf{0} & A_{2}
\end{array}\right)=G_{1} B_{1}
$$

where the so-called generator matrices $G_{1} \in K^{m \times \alpha}$ and $B_{1} \in K^{\alpha \times n}$ are rectangular, so that the displacement rank of $\mathrm{V}$ is $\leq \alpha$. As was noticed by Sakhnovich [42] (see also [43] and the relevant description of his results in [14] and [13]) in this case the Schur complement $V_{2}=V_{22}-V_{21} \frac{1}{v} V_{12}$ also has the displacement rank $\leq \alpha$, so that

$$
D_{2} V_{2}-V_{2} A_{2}=G_{2} B_{2} \text {, }
$$

for some new generator matrices $G_{2} \in K^{m \times \alpha}$ and $B_{2} \in K^{\alpha \times n}$ (also containing only $2 \alpha n$ entries). Sakhnovich was not interested in computational issues, and he used the above result for factorizing rational matrix functions. M.Morf [34] (and simultaneously [6]) independently arrived at a similar observation but they used them to derive a fast algorithm for factoring Toeplitz matrices. The technique (we suggest to call it Sakhnovich-Morf principle) is based on replacing update of $n^{2}$ elements in $V \longrightarrow V_{2}$ by updating of only $2 \alpha n$ elements in $\left\{G_{1}, B_{1}\right\} \longrightarrow\left\{G_{2}, B_{2}\right\}$. In fact they applied a divide-and-conquer technique to obtain a superfast algorithm. Their target was Toeplitz matrices but the proofs go through without much modifications for the other patterns of structure as well.

Since then a number of variants of formulas were used for computing $\left\{G_{1}, B_{1}\right\} \longrightarrow\left\{G_{2}, B_{2}\right\}$. A recent survey paper [27] can be consulted, however it covers only a subset of the relevant literature, and the reader may wish to complement it with reading recent surveys $[\mathbf{3 6}]$ and $[\mathbf{8}]$ covering more relevant literature. It is also worth to look more closely at the early references $[\mathbf{3 3}],[\mathbf{7}],[\mathbf{3 0}]$ (see also a relevant discussion in $[\mathbf{3 1}])$. 
In this paper we use one of the variants of the updating formulas $\left\{G_{1}, B_{1}\right\} \longrightarrow\left\{G_{2}, B_{2}\right\}$ obtained in $[\mathbf{1 3}]$ and $[\mathbf{1 4}]$ and recalled next.

LEMMA 2.1. Let

$$
\left(\begin{array}{cc}
D_{1} & \mathbf{0} \\
\star & D_{2}
\end{array}\right)\left(\begin{array}{ll}
V_{11} & V_{12} \\
V_{21} & V_{22}
\end{array}\right)-\left(\begin{array}{ll}
V_{11} & V_{12} \\
V_{21} & V_{22}
\end{array}\right)\left(\begin{array}{cc}
A_{1} & \star \\
\mathbf{0} & A_{2}
\end{array}\right)=\left(\frac{g_{11}}{G_{21}}\right)\left(b_{11} \mid B_{12}\right)
$$

Suppose that $v_{11}$ is nonzero. Then the Schur complement $V_{2}:=V_{22}-V_{21} V_{11}^{-1} V_{12}$ of $V$ satisfies the equation

$$
D_{2} V_{2}-V_{2} A_{2}=G_{2} B_{2}
$$

where

$$
G_{2}=G_{21}-V_{12} V_{11}^{-1} g_{11}, \quad B_{2}=B_{12}-b_{11} V_{11}^{-1} V_{12}
$$

The above lemma is a matrix part of a more general result on factorization of rational matrix functions $[\mathbf{1 3}],[\mathbf{1 4}]$. If one is focuses on this matrix part only, it admits a trivial proof (cf. with [24]).

Proof. Indeed, from (3) and the standard Schur complementation formula

$$
V=\left(\begin{array}{cc}
I & 0 \\
V_{21} V_{11}^{-1} & I
\end{array}\right)\left(\begin{array}{cc}
V_{11} & 0 \\
0 & V_{2}
\end{array}\right)\left(\begin{array}{cc}
I & V_{11}^{-1} V_{12} \\
0 & I
\end{array}\right)
$$

it follows that

$$
\begin{aligned}
& \left(\begin{array}{cc}
D_{1} & 0 \\
* & D_{2}
\end{array}\right)\left(\begin{array}{cc}
V_{11} & 0 \\
0 & V_{2}
\end{array}\right)-\left(\begin{array}{cc}
V_{11} & 0 \\
0 & V_{2}
\end{array}\right)\left(\begin{array}{cc}
A_{1} & * \\
0 & A_{2}
\end{array}\right)= \\
& \left(\begin{array}{cc}
1 & 0 \\
-V_{21} V_{11}^{-1} & I
\end{array}\right)\left(\frac{g_{11}}{G_{21}}\right)\left(b_{11} \mid B_{12}\right)\left(\begin{array}{cc}
1 & -V_{11}^{-1} V_{12} \\
0 & I
\end{array}\right) .
\end{aligned}
$$

Equating the $(2,2)$ block entries, one obtains (4).

The above simple lemma will be used to derive algorithms for processing several structured matrices appearing in the context of three decoding problems. Before addressing a number of issues on implementing the above result to solve these problems we make the following comment.

REMARK 2.2. It is obvious that the latter lemma can be used to replace the update of $n^{2}$ elements in $V \longrightarrow V_{2}$ by updating of only $2 \alpha$ n elements in $\left\{G_{1}, B_{1}\right\} \longrightarrow$ $\left\{G_{2}, B_{2}\right\}$, and to continue recursively. One can ask how should we compute the very first generator $\left\{G_{1}, B_{1}\right\}$ which is the input of the suggested algorithm. In fact, in almost all applications we know about the matrix itself is never given; what is given is a generator of this matrix. Hence no preprocessing computations are necessary. Many examples can be given, we could suggest the reader to jump to the middle of our paper and to look at the matrix in (14) appearing in the context of the Guruswami-Sudan algorithm. For this matrix its generator $\{H, U\}$ on the right hand side of (16) is immediately given by (18) and (19). So the recursive generator recursion $\{\mathrm{H}, \mathrm{U}\} \longrightarrow\left\{\mathrm{H}_{2}, \mathrm{H}_{2}\right\}$ is possible without any preprocessing computations.

Before providing the exact record of the algorithm based on lemma 2.1 we need to clarify one issue. If $V$ has a nonzero entry in its first column, say at position $(k, 1)$, then we can consider $P V$ instead of $P$, where $P$ is the matrix corresponding to interchanging rows 1 and $k$. The displacement equation for $P V$ is then given by $\left(P D P^{\top}\right)(P V)-P V A=P G B$. In order to apply the previous lemma, we need to 
ensure that $P D P^{\top}$ is lower triangular. But since $P$ can be any transposition, this implies that $D$ is a diagonal matrix.

It is possible to use the above lemma to design an algorithm for computing a $P L U$-decomposition of the matrix $V$, see [37]. Note that it is trivial to compute a nonzero element in the kernel of $V$ once a $P L U$-decomposition is known, since the kernel of $V$ and that of $U$ coincide, and a nonzero element in the latter can be found using backward substitution.

Algorithm 2.3. On input a diagonal matrix $D \in K^{m \times m}$, an upper triangular matrix $A \in K^{n \times n}$, and $a \nabla_{D, A}$-generator $(G, B)$ for $V \in K^{m \times n}$ in $D V-V A=$ $G B$, the algorithm outputs a permutation matrix $P$, a lower triangular matrix $L \in$ $K^{m \times m}$, and an upper triangular matrix $U \in K^{m \times n}$, such that $V=P L U$.

(1) Recover from the generator the first column of $V$.

(2) Determine the position, say $(k, 1)$, of a nonzero entry of $V$. If it does not exist, then set the first column of $L$ equal to $[1, \mathbf{0}]^{\top}$ and the first row of $U$ equal to the first row of $V$, and go to Step (4). Otherwise interchange the first and the $k$-th diagonal entries of $A$ and the first and the $k$-th rows of $G$ and call $P_{1}$ the permutation matrix corresponding to this transposition.

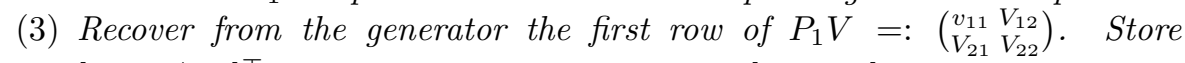
$\left[1, V_{12} / v_{11}\right]^{\top}$ as the first column of $L$ and $\left[v_{11}, V_{12}\right]$ as the first row of $U$.

(4) If $v_{11} \neq 0$, compute by Lemma 2.1 a generator of the Schur complement $V_{2}$ of $P_{1} V$. If $v_{11}=0$, then set $V_{2}:=V_{22}, G_{2}:=G_{21}$, and $B_{2}:=B_{12}$.

(5) Proceed recursively with $V_{2}$ which is now represented by its generator $\left(G_{2}, B_{2}\right)$ to finally obtain the factorization $V=P L U$, where $P=P_{1} \cdots P_{\mu}$ with $P_{k}$ being the permutation used at the $k$-th step of the recursion and $\mu=\min \{m, n\}$.

The correctness of the above algorithm and its running time depend on steps (1) and (3). Note that it may not be possible to recover the first row and column of $V$ from the matrices $D, A, G, B$. We will explore these issues later in Section 3 .

For now, we focus on developing a more memory efficient version of this algorithm for certain displacement structures. For this, the following result will be crucial.

LEMmA 2.4. Let $V \in K^{m \times n}$ be partitioned as

$$
V=\left(\begin{array}{ll}
V_{11} & V_{12} \\
V_{21} & V_{22}
\end{array}\right)
$$

for some $1 \leq i<m$, where $V_{11} \in K^{i \times i}, V_{12} \in K^{i \times(n-i)}, V_{21} \in K^{(m-i) \times i}$, and $V_{22} \in K^{(m-i) \times(m-i)}$, and suppose that $V_{11}$ is invertible. Further, let $\tilde{V}:=\left(\begin{array}{c}V \\ I_{n}\end{array}\right)$, where $I_{n}$ is the $n \times n$-identity matrix, and denote by $x=\left(x_{1}, \ldots, x_{m+n-i}\right)^{\top}$ the first column of the Schur complement of $\tilde{V}$ with respect to $V_{11}$. Suppose that $x_{1}=$ $\cdots=x_{m-i}=0$. Then the vector $\left(x_{m-i+1}, \ldots, x_{m}, 1,0, \ldots, 0\right)^{\top}$ is in the right kernel of the matrix $V$.

Proof. The Schur complement of $\tilde{V}$ with respect to $V_{11}$ is easily seen to be

$$
\left(\begin{array}{c}
V_{22}-V_{21} V_{11}^{-1} V_{12} \\
-V_{11}^{-1} V_{12} \\
I_{n-i}
\end{array}\right) \text {. }
$$


Note that

$$
V \cdot\left(\begin{array}{c}
-V_{11}^{-1} V_{12} \\
I_{n-i}
\end{array}\right)=\left(\begin{array}{c}
0 \\
V_{22}-V_{11}^{-1} V_{12}
\end{array}\right)
$$

By assumption, the first column of the matrix on the right is zero, which implies the assertion.

Suppose now that $V$ has low displacement rank with respect to $\nabla_{D, Z}$ and suppose further that $A$ is a matrix such that $A-Z$ has low rank:

$$
D V-V Z=G_{1} B_{1}, \quad A-Z=G_{2} B_{2} .
$$

Then we have (cf. with $[\mathbf{2 2}]$ )

$$
\left(\begin{array}{cc}
V & 0 \\
0 & A
\end{array}\right) \cdot\left(\begin{array}{c}
V \\
I_{n}
\end{array}\right)-\left(\begin{array}{c}
V \\
I_{n}
\end{array}\right) Z=\left(\begin{array}{cc}
G_{1} & 0 \\
0 & G_{2}
\end{array}\right) \cdot\left(\begin{array}{c}
B_{1} \\
B_{2}
\end{array}\right) .
$$

Algorithm 2.3 can now be customized in the following way.

Algorithm 2.5. On input a diagonal matrix $D \in K^{m \times m}$, an upper triangular matrix $Z \in K^{n \times n}$, a matrix $A \in K^{n \times n}$, and a $\nabla_{C, Z \text {-generator }}(G, B)$ for $W=$ $\left(\begin{array}{c}V \\ I_{n}\end{array}\right) \in K^{(m+n) \times n}$ in $C W-W Z=G B$, where $C=\left(\begin{array}{cc}D & 0 \\ 0 & A\end{array}\right)$, the algorithm outputs a vector $x=\left(x_{1}, \ldots, x_{i}, 1,0, \ldots, 0\right)^{\top}$ such that $V x=0$.

(0) Set $i:=0$.

(1) Recover from the generators the first column of $W$.

(2) Determine the position, say $(k, 1)$, of a nonzero entry of $W$. If $k$ does not exist, or $k>m-i$, then go to Step (6). Otherwise interchange the first and the $k$-th diagonal entries of $D$, the first and the $k$-th rows of $G$, the first and the $k$-th entries of the first column of $W$, denoting the new matrix by $W$ again.

(3) Recover from the generators the first row of $W$.

(4) Using the first row and the first column of $W$, compute by Lemma 2.1 a generator of the Schur complement $W_{2}$ of $W$.

(5) Proceed recursively with $V_{2}$ which is now represented by its generator $\left(G_{2}, B_{2}\right)$, increase $i$ by 1 , and go back to Step (1).

(6) Output the vector $\left(x_{1}, \ldots, x_{i}, 1,0, \ldots, 0\right)^{\top}$ where $x_{1}, \ldots, x_{i}$ are the $m-i+$ 1 -st through $m$-th entries of the first column of $W$.

As was pointed out before, the correctness of the above algorithm and its running time depend on steps (1) and (3). Note that it may not be possible to recover the first row and column of $W$ from the matrices $D, A, G, B, Z$. In fact, recovery from these data alone is only possible if $\nabla=\nabla_{C, Z}$ is an isomorphism. For simplicity we assume in the following that this is the case. In the general case one has to augment the $(D, A, G, B, Z)$ by more data corresponding to the kernel of $\nabla$, see $[\mathbf{3 6}$, Sect. 5] and Appendix A.

If $\nabla$ is an isomorphism, then the algorithm has the correct output. Indeed, using Lemma 2.1, we see that at step (5), $W_{2}$ is the Schur-complement of the matrix $P W$ with respect to the prinicipal $i \times i$-minor, where $P$ is a permutation matrix (obtained from the pivoting transpositions in Step (2)). Once the algorithm reaches Step (6), the conditions of Lemma 2.4 are satisfied and the algorithm outputs the correct vector.

The running time of the algorithm is summarized in the following. 
LEMMA 2.6. Suppose that steps (1) and (3) of Algorithm 2.5 run in time $O(\alpha m)$ and $O(\alpha n)$, respectively. Then the total running time of that algorithm is $O(\alpha m n)$, where $\alpha$ is the displacement rank of $V$ with respect to $\nabla_{D, A}$.

Proof. The proof is obvious once one realizes that Step (4) runs in time $O(\alpha(m+$ $n)$, and that the algorithm is performed recursively at most $\min \{m, n\}$ times.

\section{Computing the First Row and the First Column}

A major ingredient of Algorithm 2.5 is that of computing the first row and the first column of a matrix from its generators. The computational cost of this step depends greatly on the underlying displacement structure. In this section, we will present a solution to this problem in a special case, namely, in the case where the matrix $W \in K^{(m+n) \times n}$ has the displacement structure

$$
\left(\begin{array}{cc}
D & 0 \\
0 & A
\end{array}\right) W-W Z=G B
$$

where $D$ is diagonal and $Z, A \in K^{n \times n}$ are given by

$$
Z:=\left(\begin{array}{cccccc}
0 & 1 & 0 & \cdots & 0 & 0 \\
0 & 0 & 1 & \cdots & 0 & 0 \\
\vdots & \vdots & \vdots & \ddots & \vdots & \vdots \\
0 & 0 & 0 & \cdots & 1 & 0 \\
0 & 0 & 0 & \cdots & 0 & 1 \\
0 & 0 & 0 & \cdots & 0 & 0
\end{array}\right), \quad A:=\left(\begin{array}{cccccc}
0 & 1 & 0 & \cdots & 0 & 0 \\
0 & 0 & 1 & \cdots & 0 & 0 \\
\vdots & \vdots & \vdots & \ddots & \vdots & \vdots \\
0 & 0 & 0 & \cdots & 1 & 0 \\
0 & 0 & 0 & \cdots & 0 & 1 \\
1 & 0 & 0 & \cdots & 0 & 0
\end{array}\right) .
$$

$Z$ is called the upper shift matrix of format $n$. This case will be prototypical for our later applications. For the sake of simplicity, we will assume that all the diagonal entries of $D$ are nonzero.

Algorithm 3.1. On input a diagonal matrix $D$ with nonzero diagonal entries $x_{1}, \ldots, x_{m}$, a matrix $G=\left(G_{i j}\right) \in K^{m \times \alpha}$, and a matrix $B=\left(B_{i j}\right) \in K^{\alpha \times n}$, this algorithm computes the first row and the first column of the matrix $W \in K^{(m+n) \times n}$ which satisfies the displacement equation (6).

(1) Compute the first row $\left(r_{1}, \ldots, r_{n}\right)$ and the first column $\left(\gamma_{1}, \ldots, \gamma_{m}, \gamma_{m+1}, \ldots, \gamma_{m+n}\right)^{\top}$ of $G B$

(2) For $i=1, \ldots, m$ set $v_{i}:=\gamma_{i} / x_{i}$.

(3) For $i=m+1, \ldots, m+n-1$ set $v_{i+1}:=\gamma_{i}$.

(4) Set $v_{m+1}:=\gamma_{m+n}$.

(5) Set $c_{1}:=v_{1}$. For $i=2, \ldots, n$ set $c_{i}:=\left(r_{i}+c_{i-1}\right) / x_{i}$.

(4) Output row $\left(c_{1}, \ldots, c_{n}\right)$ and column $\left(v_{1}, \ldots, v_{m}, v_{m+1}, \ldots, v_{m+n}\right)^{\top}$.

Proposition 3.2. The above algorithm correctly computes its output with $O(\alpha(m+n))$ operations in the field $K$.

ProOF. Correctness of the algorithm follows immediately from the following observation: let $\left(c_{1}, \ldots, c_{n}\right)$ and $\left(c_{1}, v_{2}, \ldots, v_{m}, v_{m+1}, \ldots, v_{m+n}\right)^{\top}$ be the first row 
and the first column of $W$, respectively. Then

$$
\left(\begin{array}{cc}
D & 0 \\
0 & A
\end{array}\right) W-W Z=\left(\begin{array}{cccc}
x_{1} c_{1} & x_{1} c_{2}-c_{1} & \cdots & x_{1} c_{n}-c_{n-1} \\
x_{2} v_{2} & \star & \cdots & \star \\
\vdots & \vdots & \ddots & \vdots \\
x_{m} v_{m} & \star & \cdots & \star \\
v_{m+2} & \star & \cdots & \star \\
\vdots & \vdots & \cdots & \vdots \\
v_{m+n} & \star & \ddots & \star \\
v_{m+1} & \star & \cdots & \star
\end{array}\right) \text {, }
$$

where the $\star$ 's denote elements in $K$. As for the running time, observe first that computing the first row and first column of $G B$ requires at most $\alpha(m+n)+\alpha n$ operations over $K$. Step (2) can be done using $m$ operations, steps (3) and (4) are free of charge, and Step (5) requires $2 n$ operations.

We can now combine all the results obtained so far to develop an efficient algorithm for computing a nonzero element in the kernel of a matrix $V \in K^{m \times n}$ given indirectly as a solution to the displacement equation

$$
D V-V Z=G B \text {. }
$$

Here, $D$ is a diagonal matrix, $Z$ is an upper-shift matrix of format $n, G \in K^{m \times \alpha}$, and $B \in K^{\alpha \times n}$. Since we will use Algorithm 2.5, we need to have a displacement structure for $W=\left(\begin{array}{c}V \\ I_{n}\end{array}\right)$. It is given by

$$
\left(\begin{array}{cc}
D & 0 \\
0 & A
\end{array}\right)\left(\begin{array}{c}
V \\
I_{n}
\end{array}\right)-\left(\begin{array}{c}
V \\
I_{n}
\end{array}\right) Z=\left(\begin{array}{c}
G \\
C
\end{array}\right) B
$$

where $A$ is defined as in (7) and $C=A-Z$. We assume that none of the diagonal entries of $D$ are zero.

Algorithm 3.3. On input integers $m, n, m<n$, a diagonal matrix $D \in K^{m \times m}$ all of whose diagonal entries $x_{1}, \ldots, x_{m}$ are nonzero, a matrix $G \in K^{m \times \alpha}$, and a matrix $B \in K^{\alpha \times n}$, the algorithm computes a nonzero vector $v \in K^{n}$ in the kernel of the matrix $V$ given by the displacement equation (8).

(1) Set $G_{1}:=\left(\begin{array}{c}G \\ A-Z\end{array}\right), B_{1}:=B$, and $D_{1}:=D$, where $A$ is given in (7).

(2) For $i=1, \ldots, m$ do

(a) Let $D_{i}$ be the diagonal matrix with diagonal entries $x_{i}, \ldots, x_{m}$. Use Algorithm 3.1 with input $D_{i}, G_{i}$, and $B_{i}$ to compute the column $c=\left(c_{1}, \ldots, c_{m+n-i+1}\right)^{\top}$.

(b) If $c_{1}=\cdots=c_{m-i+1}=0$, then output $\left(c_{m-i+2}, \ldots, c_{n}, 1,0, \ldots, 0\right)^{\top}$ and stop. Otherwise, find an integer $k \leq m-i+1$ such that $c_{k} \neq 0$. Interchange rows $k$ and 1 of $c$ and of $G_{i}$, interchange the first and the kth diagonal entries of $D_{i}$.

(c) Use Algorithm 3.1 with input $D_{i}, G_{i}$ and $B_{i}$ to compute the row $r=\left(r_{1}, \ldots, r_{n-i+1}\right)$.

(d) For $j=2, \ldots, m+n-i+1$ replace row $j$ of $G_{i}$ by $-c_{j} / c_{1}$ times the first row plus the $j$-th row. Set $G_{i+1}$ as the matrix formed from $G_{i}$ by deleting the first row. 
(e) For $j=2, \ldots, n-i+1$ replace the $j$-th column of $B_{i}$ by $-r_{j} / c_{1}$ times the first column plus the $j$-th column. Set $B_{i+1}$ as the matrix formed from $B_{i}$ by deleting the first column.

THEOREM 3.4. The above algorithm correctly computes its output with $O(\alpha m n)$ operations over the field $K$.

Proof. The correctness of the algorithm follows from Lemma 2.4 and Proposition 3.2. The analysis of the running time of the algorithm is straight-forward: Step (2a) runs in time $O(\alpha(m+n-i))$ by Proposition 3.2. The same is true for steps $(2 \mathrm{~d})$ and $(2 \mathrm{e})$. Hence, in the worst case, the running time will be $\sum_{i=1}^{m-1} O(\alpha(m+n-i+1))=O(\alpha m n)$, since $m<n$.

The memory requirements for this algorithm can be reduced by observing that the last $n-i$ rows of the matrix $G_{i}$ are always known. As a result, the matrix $G_{i}$ needs to store only $m(\ell+1)$ elements (instead of $(m+n)(\ell+1)$ ). Further, it is easy to see that for the vector $c$ computed in Step (2a), we have $c_{m+1}=1, c_{m+2}=$ $\cdots=c_{m+n-i}=0$, hence, we do not need to store these results. Combining these observations, one can easily design an algorithm that needs storage for two matrices of sizes $m \times(\ell+1)$ and $(\ell+1) \times n$, respectively, and storage for three vectors of size $n$.

\section{The Algorithm of Sudan}

In [45] Sudan describes an algorithm for list decoding of RS-codes which we will briefly describe here. Let $\mathbb{F}_{q}[x]_{<k}$ denote the space of polynomials over the finite field $\mathbb{F}_{q}$ of degree less than $k$, and let $x_{1}, \ldots, x_{n}$ denote distinct elements of $\mathbb{F}_{q}$, where $k \leq n$. The image of the morphism $\gamma: \mathbb{F}_{q}[x]_{<k} \rightarrow \mathbb{F}_{q}^{n}$ mapping a polynomial $f$ to the vector $v:=\left(f\left(x_{1}\right), \ldots, f\left(x_{n}\right)\right)$ is a linear code over $\mathbb{F}_{q}$ of dimension $k$ and minimum distance $n-k+1$. Suppose the vector $v$ is sent over a communication channel and the vector $u:=\left(y_{1}, \ldots, y_{n}\right)$ is received. If the Hamming distance between $u$ and $v$ is at most $(n-k) / 2$, then conventional decoding algorithms like the Berlekamp-Massey algorithm can decode $u$ to the correct codeword $v$. If the number $e$ of errors is larger than $(n-k) / 2$, then Sudan's algorithm compiles a list of at most $\ell=\ell(e)$ codewords which contains $v$. The algorithm consists of two steps. Let $b:=\lfloor n /(\ell+1)+\ell(k-1) / 2+1\rfloor$. The first step uses Lagrange interpolation to compute a bivariate polynomial $F=\sum_{i=0}^{\ell} F_{i}(x) y^{i}$ with $\operatorname{deg} F_{i}<b-i(k-1)$ and such that $F\left(x_{t}, y_{t}\right)=0$ for all $t=1, \ldots, n$. The second step computes the roots of $F$ which are of the form $y-g(x), g \in \mathbb{F}_{q}[x]_{<k}$, and outputs those $g$ such that $\gamma(g)$ and $u$ have distance at most $e$. The relationship between $\ell$ and $e$ is given indirectly by $e \leq n-b$. There are efficient algorithms for solving the second step $[\mathbf{2 , 1 1 , 3 9 ]}$. In the following we will concentrate on the problem of efficiently computing the polynomial $F$.

This polynomial corresponds to a nonzero element in the kernel of a matrix $V$ with a repetitive structure which we will describe below. Let $d_{0}, \ldots, d_{\ell} \geq 0$ be 
defined by $d_{i}:=b-i(k-1)$ for $0 \leq i<\ell$, and $d_{\ell}=n+1-\sum_{i=0}^{\ell-1} d_{i}$. Let $V:=$ (10)

$$
\left(\begin{array}{ccccccccccccc}
1 & x_{1} & \cdots & x_{1}^{d_{0}-1} & y_{1} & y_{1} x_{1} & \cdots & y_{1} x_{1}^{d_{1}-1} & \cdots & y_{1}^{\ell} & y_{1}^{\ell} x_{1} & \cdots & y_{1}^{\ell} x_{1}^{d_{\ell}-1} \\
1 & x_{2} & \cdots & x_{2}^{d_{0}-1} & y_{2} & y_{2} x_{2} & \cdots & y_{2} x_{2}^{d_{1}-1} & \cdots & y_{2}^{\ell} & y_{2}^{\ell} x_{2} & \cdots & y_{2}^{\ell} x_{2}^{d_{\ell}-1} \\
\vdots & \vdots & \ddots & \vdots & \vdots & \vdots & \ddots & \vdots & \ddots & \vdots & \vdots & \ddots & \vdots \\
1 & x_{n} & \cdots & x_{n}^{d_{0}-1} & y_{n} & y_{n} x_{n} & \cdots & y_{n} x_{n}^{d_{1}-1} & \cdots & y_{n}^{\ell} & y_{n}^{\ell} x_{n} & \cdots & y_{n}^{\ell} x_{n}^{d_{\ell}-1}
\end{array}\right),
$$

and let $v=\left(v_{00}, v_{01}, \ldots, v_{0, d_{0}-1}, \ldots, v_{\ell, 0}, v_{\ell, 1}, \ldots, v_{\ell, d_{\ell}-1}\right) \in \mathbb{F}_{q}^{n+1}$ be a nonzero vector such that $V \cdot v=0$. Then the polynomial $F(x, y)=\sum_{i=0}^{\ell} F_{i}(x) y^{i}$ where $F_{i}(x)=v_{i, 0}+v_{i, 1} x+\cdots+v_{i, d_{i}-1} x^{d_{i}-1}$ satisfies $F\left(x_{t}, y_{t}\right)=0$ for $t=1, \ldots, n$. The task at hand is thus to compute a nonzero element in the kernel of $V$. Using the displacement approach we can easily compute such a vector in time $O\left(n^{2} \ell\right)$. For constant list-size $\ell$ we thus obtain an algorithm whose running time is quadratic in $n$.

Let us first prove that $V$ has displacement rank at most $\ell+1$ with respect to suitable matrices. Let $D$ be the diagonal matrix with diagonal entries $1 / x_{1}, \ldots, 1 / x_{n}$. Define

$$
G:=\left(\begin{array}{cccc}
1 / x_{1} & y_{1} / x_{1}-x_{1}^{d_{0}-1} & \cdots & y_{1}^{\ell-1}\left(y_{1} / x_{1}-x_{1}^{d_{\ell-1}-1}\right) \\
1 / x_{2} & y_{2} / x_{2}-x_{2}^{d_{0}-1} & \cdots & y_{2}^{r-2}\left(y_{2} / x_{2}-x_{2}^{d_{\ell-1}-1}\right) \\
\vdots & \vdots & \ddots & \vdots \\
1 / x_{n} & y_{n} / x_{n}-x_{n}^{d_{0}-1} & \cdots & y_{n}^{r-2}\left(y_{n} / x_{n}-x_{n}^{d_{\ell-1}-1}\right)
\end{array}\right)
$$

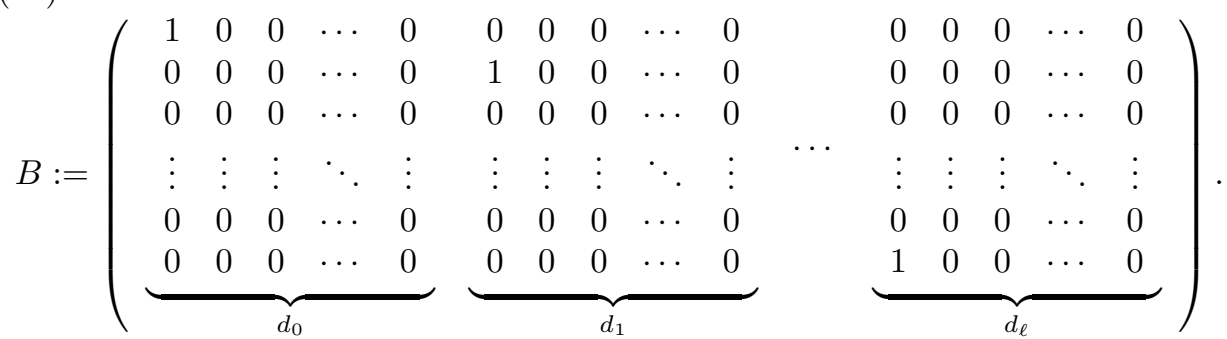

Then, one easily proves that

$$
D V-V Z=G B
$$

where $Z$ is the upper shift matrix of format $n+1$ defined in (7). Hence, $V$ has displacement rank at most $\ell+1$ with respect to $\nabla_{D, Z}$, and we can use Algorithm 3.3 to compute a nonzero element in the kernel of $V$.

Algorithm 4.1. On input $\left(x_{1}, y_{1}\right), \ldots,\left(x_{n}, y_{n}\right) \in \mathbb{F}_{q}^{2}$ and integers $d_{0}, d_{1}, \ldots, d_{\ell}$ such that $\sum_{i=0}^{\ell} d_{i}=n+1$ and such that none of the $x_{i}$ is zero, this algorithm computes a a polynomial $F(x, y)=\sum_{i=0}^{\ell} F_{i}(x) y^{i}$ such that $F\left(x_{i}, y_{i}\right)=0$ for $i=$ $1, \ldots, n$.

(1) Run Algorithm 3.3 on input $D, G, B$, where $D$ is the diagonal matrix with entries $1 / x_{1}, \ldots, 1 / x_{n}$, and $G, B$ given in (11) and (12), respectively. Let $v=\left(v_{00}, v_{01}, \ldots, v_{0, d_{0}-1}, \ldots, v_{\ell, 0}, v_{\ell, 1}, \ldots, v_{\ell, d_{\ell}-1}\right)$ denote the output.

(2) Output $F(x, y)=\sum_{i=0}^{\ell} F_{i}(x) y^{i}$ where $F_{i}(x)=v_{i, 0}+v_{i, 1} x+\cdots+$ $v_{i, d_{i}-1} x^{d_{i}-1}$. 
Theorem 3.4 immediately implies the following result.

THEOREM 4.2. Algorithm 4.1 correctly computes its output with $O\left(n^{2} \ell\right)$ operations over the field $K$.

There are various methods to extend the algorithm to the case where one of the $x_{i}$ is zero. We will sketch one of them. Without loss of generality, assume that $x_{1}=0$. In this case, deletion of the first row of the matrix $V$ yields another matrix $\tilde{V}$ of the form given in (10) in which none of the $x_{i}$ is zero. The matrix $V$ has then the following displacement structure

$$
\left(\begin{array}{ccccc}
1 & 0 & 0 & \cdots & 0 \\
0 & 1 / x_{2} & 0 & \cdots & 0 \\
\vdots & \vdots & \vdots & \ddots & \vdots \\
0 & 0 & 0 & \cdots & 1 / x_{n}
\end{array}\right) V-V Z=\left(\begin{array}{cc}
0_{1 \times(\ell+1)} & 1 \\
G & 0_{(n-1) \times 1}
\end{array}\right) \cdot\left(\begin{array}{c}
B \\
x
\end{array}\right)
$$

where $x$ is the vector

$$
x=(\underbrace{1,-1,0, \ldots, 0}_{d_{0}}, \underbrace{y_{1},-y_{1}, 0, \ldots, 0}_{d_{1}}, \cdots, \underbrace{y_{1}^{\ell},-y_{1}^{\ell}, 0, \ldots, 0}_{d_{\ell}}),
$$

$G$ is the matrix obtained from the one given in (11) by deleting the first row, and $0_{a \times b}$ denotes the $a \times b$-matrix consisting of zeros. We can now run Algorithm 3.3 on the new set of matrices to obtain a nonzero element in the kernel of $V$.

We finish this section with an example. Suppose that $C$ is the Reed-Solomon code of dimension 4 given as the set of evaluations of polynomials of degree at most 3 over the field $\mathbb{F}_{31}$ at the points $x_{1}=1, x_{2}=2, \ldots, x_{30}=30$. This code can correct up to 15 errors with lists of size at most 2 . In this case, $\ell=2, n=30$, $k=4, b=\lfloor n /(\ell+1)+\ell k / 2+1\rfloor=14, d_{0}=14, d_{1}=11$, and $d_{2}=8$.

Suppose that we have received the vector

$$
y=(3,13,0,6,7,24,19,25,1,17,19,5,10,0,19,2,4,23,28,23,29,7,8,12,27,24,15,6,22,30)
$$

Then the matrix $G$ given in (11) is as follows $G^{\top}=$

$\left(\begin{array}{cccccccccccccccccccccccccccccc}1 & 16 & 21 & 8 & 25 & 26 & 9 & 4 & 7 & 28 & 17 & 13 & 12 & 20 & 29 & 2 & 11 & 19 & 18 & 14 & 3 & 24 & 27 & 22 & 5 & 6 & 23 & 10 & 15 & 30 \\ 2 & 14 & 7 & 15 & 15 & 29 & 28 & 22 & 20 & 2 & 23 & 17 & 16 & 3 & 28 & 0 & 10 & 14 & 25 & 2 & 3 & 0 & 15 & 4 & 17 & 25 & 6 & 22 & 28 & 2 \\ 6 & 25 & 0 & 3 & 12 & 23 & 15 & 26 & 2 & 9 & 28 & 14 & 3 & 0 & 3 & 6 & 14 & 16 & 20 & 6 & 22 & 25 & 15 & 16 & 25 & 19 & 14 & 24 & 15 & 0\end{array}\right)$

The matrix $B$ in (12) is as follows:

$B=\left(\begin{array}{lllllllllllllllllllllllllllllll}\mathbf{1} & 0 & 0 & 0 & 0 & 0 & 0 & 0 & 0 & 0 & 0 & 0 & 0 & 0 & 0 & 0 & 0 & 0 & 0 & 0 & 0 & 0 & 0 & 0 & 0 & 0 & 0 & 0 & 0 & 0 & 0 \\ 0 & 0 & 0 & 0 & 0 & 0 & 0 & 0 & 0 & 0 & 0 & 0 & 0 & 0 & \mathbf{1} & 0 & 0 & 0 & 0 & 0 & 0 & 0 & 0 & 0 & 0 & 0 & 0 & 0 & 0 & 0 & 0 \\ 0 & 0 & 0 & 0 & 0 & 0 & 0 & 0 & 0 & 0 & 0 & 0 & 0 & 0 & 0 & 0 & 0 & 0 & 0 & 0 & 0 & 0 & 0 & 0 & 0 & 1 & 0 & 0 & 0 & 0 & 0\end{array}\right)$.

The matrix $D$ of Algorithm 4.1 is the diagonal matrix with diagonal entries

$(1,16,21,8,25,26,9,4,7,28,17,13,12,20,29,2,11,19,18,14,3,24,27,22,5,6,23,10,15,30)$.

In the first round of Algorithm 3.3, the first column computed in step 2(a) is

$$
(1,1,1,1,1,1,1,1,1,1,1,1,1,1,1,1,1,1,1,1,1,1,1,1,1,1,1,1,1,1)^{\top} \text {. }
$$

The first row, computed at step $2(\mathrm{c})$, is

$$
(1,1,1,1,1,1,1,1,1,1,1,1,1,1,3,3,3,3,3,3,3,3,3,3,3,9,9,9,9,9,9) .
$$

The loop in step 2 of Algorithm 3.3 is performed 29 times. The kernel element computed at the end equals

$(12,5,4,9,20,19,28,26,23,18,11,16,14,25,26,15,14,25,1,25,29,27,9,17,6,24,30,7,20,1,0)$. 
It corresponds to the bivariate polynomial $F(x, y)=$

$$
\begin{aligned}
& \left(12+5 x+4 x^{2}+9 x^{3}+20 x^{4}+19 x^{5}+28 x^{6}+26 x^{7}+23 x^{8}+18 x^{9}+11 x^{10}\right. \\
& \left.+16 x^{11}+14 x^{12}+25 x^{13}\right)+ \\
& \left(26+15 x+14 x^{2}+25 x^{3}+x^{4}+25 x^{5}+29 x^{6}+27 x^{7}+9 x^{8}+17 x^{9}+6 x^{10}\right) y+ \\
& \left(24+30 x+7 x^{2}+20 x^{3}+x^{4}\right) y^{2} .
\end{aligned}
$$

Applying the algorithm of, e.g., [11], we obtain

$F(x, y) \equiv\left(y-\left(1+x+x^{3}\right)\right)\left((y-16)+(9 y-1) x+(6-y) x^{2}+(29+6 y) x^{3}+(22 y+28) x^{4}\right) \bmod x^{5}$,

which shows that there is at most one polynomial, $f(x)=1+x+x^{3}$, which satisfies $F(x, f(x))=0$. A further calculation shows that this is indeed the case. Hence, there is only one codeword in $C$ of distance at most 15 from the received word $y$, namely the codeword corresponding to $f$.

\section{The Algorithm of Shokrollahi-Wasserman}

In [44] the authors generalize Sudan's algorithm to algebraic-geometric (AG-) codes. We briefly discuss this generalization. Let $\mathcal{X}$ be an irreducible algebraic curve over the finite field $\mathbb{F}_{q}$, let $Q, P_{1}, \ldots, P_{n}$ be distinct $\mathbb{F}_{q}$-rational points of $\mathcal{X}$, and let $L(\alpha Q)$ denote the linear space of the divisor $\alpha Q$, i.e., the space generated by all functions in the function field of $\mathcal{X}$ that have only a pole of order at most $\alpha$ at $Q$. The (one-point) AG-code associated to these data is then defined as the image of the $\mathbb{F}_{q}$-linear map $L(\alpha Q) \rightarrow \mathbb{F}_{q}^{n}$ mapping a function $f$ to the vector $\left(f\left(P_{1}\right), \ldots, f\left(P_{n}\right)\right)$. It is well known that this linear code has dimension $k \geq \alpha-g+1$ and minimum distance $d \geq n-\alpha$, where $g$ denotes the genus of the curve. Suppose that we want to decode this code with a list of length at most $\ell$. Let $\beta:=\lfloor(n+1) /(\ell+1)+\ell \alpha / 2+g\rfloor$. Let $\varphi_{1}, \ldots, \varphi_{t}, t=\beta-g+1$, be elements of $L(\beta Q)$ with strictly increasing pole orders at $Q$. Let $\left(y_{1}, \ldots, y_{n}\right)$ be the received word. The algorithm in [44] first finds functions $u_{0}, \ldots, u_{\ell}$ with $u_{i} \in L((\beta-i \alpha) Q)$ such that $\sum_{i} u_{i}\left(P_{j}\right) y_{j}^{i}=0$ for all $1 \leq j \leq n$. This step is accomplished by computing a nonzero element in the kernel of the matrix

$$
V:=\left(\begin{array}{llll}
V_{0} & V_{1} & \ldots & V_{l}
\end{array}\right)
$$

with

$$
\begin{gathered}
V_{0}=\left(\begin{array}{ccc}
\varphi_{1}\left(P_{1}\right) & \cdots & \varphi_{s_{0}}\left(P_{1}\right) \\
\varphi_{1}\left(P_{2}\right) & \cdots & \varphi_{s_{0}}\left(P_{2}\right) \\
\vdots & \ddots & \vdots \\
\varphi_{1}\left(P_{n}\right) & \cdots & \varphi_{s_{0}}\left(P_{n}\right)
\end{array}\right), \\
V_{1}=\left(\begin{array}{ccc}
y_{1} \varphi_{1}\left(P_{1}\right) & \cdots & y_{1} \varphi_{s_{1}}\left(P_{1}\right) \\
y_{2} \varphi_{1}\left(P_{2}\right) & \cdots & y_{2} \varphi_{s_{1}}\left(P_{2}\right) \\
\vdots & \ddots & \vdots \\
y_{n} \varphi_{1}\left(P_{n}\right) & \cdots & y_{n} \varphi_{s_{1}}\left(P_{n}\right)
\end{array}\right), \quad V_{l}=\left(\begin{array}{ccc}
y_{1}^{\ell} \varphi_{1}\left(P_{1}\right) & \cdots & y_{1}^{\ell} \varphi_{s_{\ell}}\left(P_{1}\right) \\
y_{2}^{\ell} \varphi_{1}\left(P_{2}\right) & \cdots & y_{2}^{\ell} \varphi_{s_{\ell}}\left(P_{2}\right) \\
\vdots & \ddots & \vdots \\
y_{n}^{\ell} \varphi_{1}\left(P_{n}\right) & \cdots & y_{n}^{\ell} \varphi_{s_{\ell}}\left(P_{n}\right)
\end{array}\right),
\end{gathered}
$$

where $s_{j}=\beta-j \alpha-g+1$ for $j<\ell$ and $\sum_{i=0}^{\ell}\left(s_{j}+1\right)=n+1$. To simplify the discussions, we assume that there are two functions $\varphi, \psi \in L(\beta Q)$ such that all the $\varphi_{i}$ are of the form $\varphi^{a} \psi^{b}$ and such that the order of poles at $Q$ of $\varphi$ is smaller than that of $\psi$. Further, we assume that $\varphi$ does not vanish at any of the points $P_{1}, \ldots, P_{n}$. We remark that the method described below can be modified to deal with a situation in which any of the above assumptions is not valid. 
Let $d$ be the order of poles of $\varphi$ at $Q$. Then it is easily seen that any element of $L(\beta Q)$ is of the form $\varphi^{a} \psi^{b}$, where $0 \leq b<d$. Now we divide each of the blocks of the matrix $V$ into subblocks in the following way: by changing the order of the columns, we write the $t$-th block of $V$ in the form

with

$$
V_{t}=\left(\begin{array}{lll}
V_{t, 0} & \ldots & V_{t, s}
\end{array}\right)
$$

and

$$
V_{t, 0}=\left(\begin{array}{ccc}
\varphi\left(P_{1}\right)^{0} \psi\left(P_{1}\right)^{0} y_{1}^{t} & \cdots & \varphi\left(P_{1}\right)^{a_{0}} \psi\left(P_{1}\right)^{0} y_{1}^{t} \\
\varphi\left(P_{2}\right)^{0} \psi\left(P_{2}\right)^{0} y_{2}^{t} & \cdots & \varphi\left(P_{2}\right)^{a_{0}} \psi\left(P_{2}\right)^{0} y_{2}^{t} \\
\vdots & \ddots & \vdots \\
\varphi\left(P_{n}\right)^{0} \psi\left(P_{n}\right)^{0} y_{n}^{t} & \cdots & \varphi\left(P_{n}\right)^{a_{0}} \psi\left(P_{n}\right)^{0} y_{n}^{t}
\end{array}\right)
$$

$$
V_{t, s}=\left(\begin{array}{ccc}
\varphi\left(P_{1}\right)^{0} \psi\left(P_{1}\right)^{s} y_{1}^{t} & \cdots & \varphi\left(P_{1}\right)^{a_{s}} \psi\left(P_{1}\right)^{s} y_{1}^{t} \\
\varphi\left(P_{2}\right)^{0} \psi\left(P_{2}\right)^{s} y_{2}^{t} & \cdots & \varphi\left(P_{2}\right)^{a_{s}} \psi\left(P_{2}\right)^{s} y_{2}^{t} \\
\vdots & \ddots & \vdots \\
\varphi\left(P_{n}\right)^{0} \psi\left(P_{n}\right)^{s} y_{n}^{t} & \cdots & \varphi\left(P_{n}\right)^{a_{s}} \psi\left(P_{n}\right)^{s} y_{n}^{t}
\end{array}\right)
$$

By the above remarks, $s<d$. Let now $D$ be the diagonal matrix $\operatorname{diag}[\varphi]_{1, n}$, and let $Z$ be the upper shift matrix of format $n+1$. Then in a similar way as in the last section one can construct a matrix $G \in \mathbb{F}_{q}^{n \times d \ell}$ and a matrix $B \in \mathbb{F}_{q}^{d \ell \times m}$ such that $D V-V Z=G B$, where $Z$ is the upper shift matrix of format $n+1$. Using Algorithm 3.3 we then obtain the following result.

THEOREM 5.1. Using Algorithm 3.3, one can compute a nonzero element in the kernel of the matrix $V$ of (13) with $O\left(n^{2} d \ell\right)$ operations over the base field.

A final estimate of the running time of this algorithm has to take into account the relationship between $d$ and $n$. This depends on the specific structure of the curve and the divisor used in the definition. For instance, if the curve has a nonsingular plane model of the form $G(x, y)=0$, and $G$ is a monic polynomial of degree $d$ in the variable $y$, and if we choose for $Q$ the common pole of the functions $x$ and $y$, then the above conditions are all valid (see also $[\mathbf{2 0}]$ ). As an example, consider the case of a Hermitian curve defined over $\mathbb{F}_{q^{2}}$ by the equation $x^{q+1}=y^{q}+y$. In this case, the parameter $d$ equals $q+1$, which $n=q^{3}$. As a result, the algorithm uses $O\left(n^{7 / 3} \ell\right) \mathbb{F}_{q}$-operations. Note that for $\ell=1$, i.e., for unique decoding, this is exactly the running time of the algorithm presented in [20].

We remark that there are plenty of other fast algorithms for (conventional) decoding of AG-codes which make use of the structure of the matrices, though in a more implicit form than given here, see, e.g., $[\mathbf{1 9}]$ and the references therein, or the work [28].

\section{The Algorithm of Guruswami-Sudan}

In $[\mathbf{1 5}]$ the authors describe an extension of algorithms presented in [45] and [44] in which they use a variant of Hermite interpolation rather than a Lagrange interpolation. In the case of Reed-Solomon codes, the input to the algorithm is a set of $n$ points $\left(x_{i}, y_{i}\right)$ in the affine plane over $\mathbb{F}_{q}$, and parameters $r, k$, and $\ell$. Let $\beta$ be the smallest integer such that $(\ell+1) \beta>\left(\begin{array}{c}\ell+1 \\ 2\end{array}\right) k+\left(\begin{array}{c}r+1 \\ 2\end{array}\right) n$. The output of the algorithm is a nonzero bivariate polynomial $G=\sum_{i=0}^{\ell} G_{i}(x) y^{i}$ such that $\operatorname{deg}\left(G_{i}\right)<\beta-i(k-1)$, $G\left(x_{i}, y_{i}\right)=0$ for all $i \leq n$, and $G\left(x+x_{i}, y+y_{i}\right)$ does not contain any monomial of 
degree less than $r$. Such a polynomial $G$ corresponds to a nonzero element in the kernel of a certain matrix $V$ which we will describe below. Let $t \leq n$ be a fixed positive integer. For $0 \leq i<r$ and $0 \leq j \leq \ell$ let $V_{i j}^{t} \in \mathbb{F}_{q}^{(r-i) \times(\beta-j(k-1))}$ be the matrix having $(\mu, \nu)$-entry equal to $\left(\begin{array}{l}\nu \\ \mu\end{array}\right) x_{t}^{\nu-\mu}$, where $0 \leq \mu<r-i$ and $0 \leq \nu<\beta-j(k-1)$. Now define the matrix $V_{t}$ as a block matrix with $r$ block rows and $\ell+1$ block columns, with block entry $(i, j)$ equal to $\left(\begin{array}{l}j \\ i\end{array}\right) y_{t}^{j-i} V_{i j}^{t}$. The matrix $V$ then equals

$$
V=\left(\begin{array}{c}
V_{1} \\
V_{2} \\
\vdots \\
V_{n}
\end{array}\right)
$$

$V$ has $m:=\left(\begin{array}{c}r+1 \\ 2\end{array}\right) n$ rows and $s:=(\ell+1) \beta-\left(\begin{array}{c}\ell+1 \\ 2\end{array}\right)(k-1)$ columns (and $\left.s>m\right)$. In the following we will show that $V$ has a displacement structure with respect to suitable matrices.

Let $J_{i}^{t}$ denote the $i \times i$-Jordan block having $x_{t}$ in its main diagonal and 1 's on its lower sub-diagonal. Let $J^{t}$ be the block diagonal matrix with block diagonal entries $J_{r}^{t}, \ldots, J_{1}^{t}$. Let $J$ be the block diagonal matrix with block diagonal entries $J^{1}, \ldots, J^{n}$ :

$$
J:=\underbrace{\left(\begin{array}{cccc}
J^{1} & 0 & \cdots & 0 \\
0 & J^{2} & \cdots & 0 \\
\vdots & \vdots & \ddots & \vdots \\
0 & 0 & \cdots & J^{n}
\end{array}\right)}_{n\left(\begin{array}{c}
r+1 \\
2
\end{array}\right)}
$$

with

$$
J^{t}:=\underbrace{\left(\begin{array}{cccc}
J_{r}^{t} & 0 & \cdots & 0 \\
0 & J_{r-1}^{t} & \cdots & 0 \\
\vdots & \vdots & \ddots & \vdots \\
0 & 0 & \cdots & J_{1}^{t}
\end{array}\right)}_{\left(\begin{array}{ccc}
r_{2}+1 \\
2
\end{array}\right)}, J_{i}^{t}:=\underbrace{\left(\begin{array}{cccccc}
x_{t} & 0 & 0 & \cdots & 0 & 0 \\
1 & x_{t} & 0 & \cdots & 0 & 0 \\
0 & 1 & x_{t} & \cdots & 0 & 0 \\
\vdots & \vdots & \vdots & \ddots & \vdots & \vdots \\
0 & 0 & 0 & \cdots & 1 & x_{t}
\end{array}\right)}_{i} .
$$

Then, a quick calculation shows that

$$
J V-V Z_{s}^{\top}=H U
$$


where $Z_{s}$ is the upper shift matrix of format $s$, and $H$ and $U$ are described as follows: for $1 \leq t \leq n$ let $H_{t}=\in \mathbb{F}_{q}^{\left(\begin{array}{c}r+1 \\ 2\end{array}\right) \times(\ell+1)}$ be given by

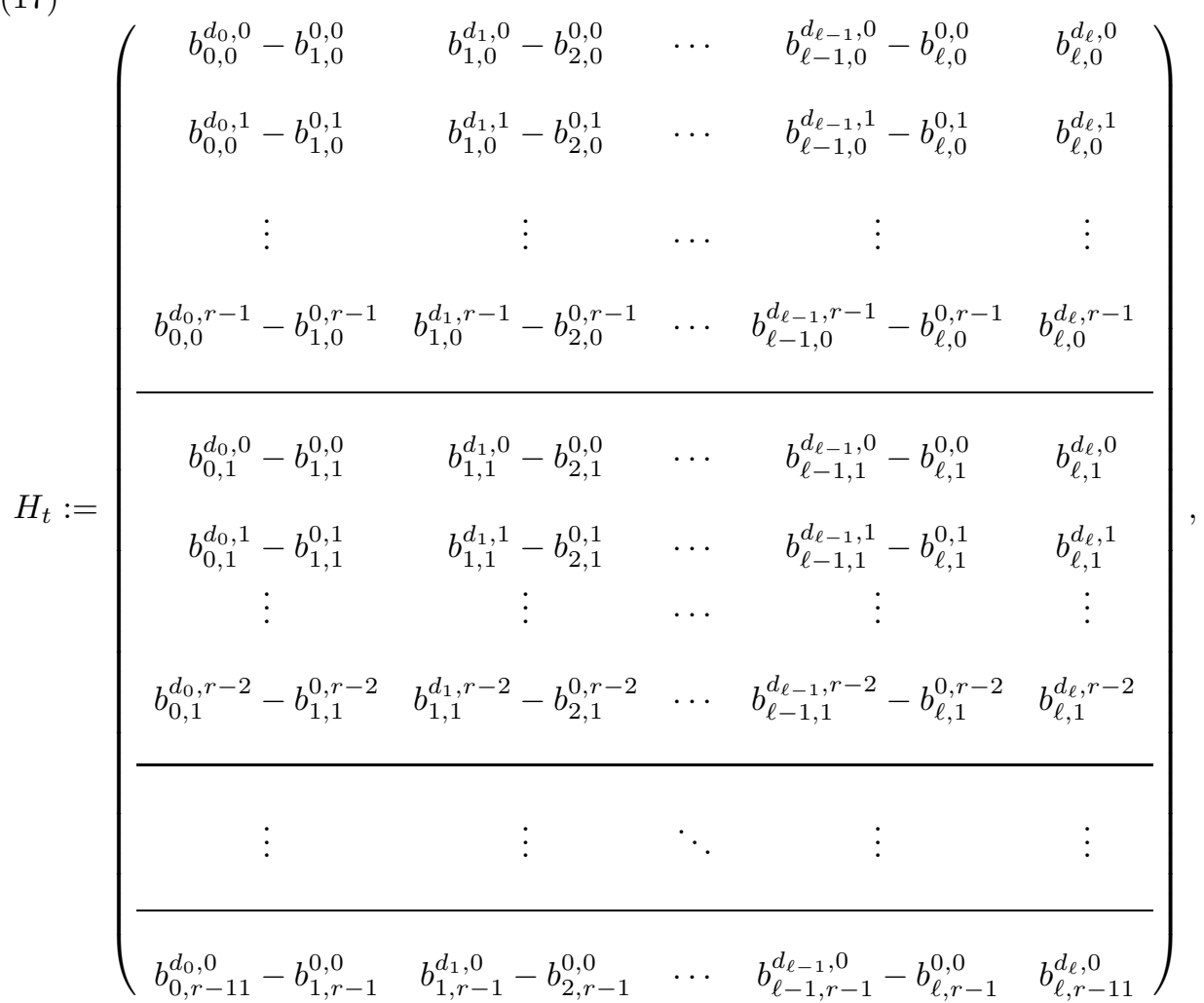

where $b_{c, d}^{e, f}:=\left(\begin{array}{l}c \\ d\end{array}\right)\left(\begin{array}{l}e \\ f\end{array}\right) y_{t}^{c-d} x_{t}^{e-f}$. Then

$$
H=\left(\begin{array}{c}
H_{1} \\
H_{2} \\
\vdots \\
H_{n}
\end{array}\right),
$$

and

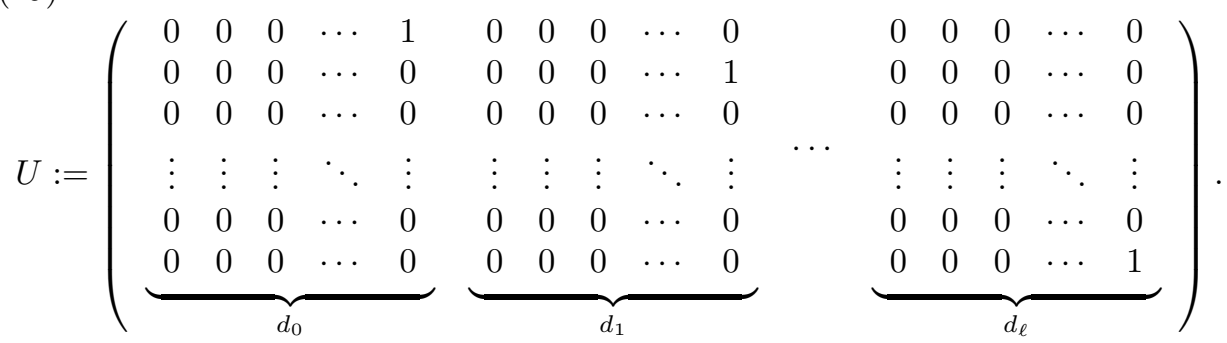

Unfortunately, we cannot apply the results of Section 2 to this situation directly, since $J$ is not a diagonal matrix and $Z_{s}^{\top}$ is not upper triangular. A remedy of the situation is as follows. Let $\mathbb{F}$ be a suitable extension of $\mathbb{F}_{q}$ which contains $s$ nonzero pairwise distinct elements $\epsilon_{1}, \ldots, \epsilon_{s}$. Let $W$ be the $s \times s$-Vandermonde 
matrix having $(i, j)$-entry $\epsilon_{i}^{j-1}$ and let $\Delta$ be the diagonal matrix with diagonal entries $1 / \epsilon_{1}, \ldots, 1 / \epsilon_{s}$. Then we have the following displacement structure for $W$

$$
\Delta W-W Z=E F,
$$

where $E=\left(1 / \epsilon_{1}, 1 / \epsilon_{2}, \ldots, 1 / \epsilon_{s}\right)^{\top}$ and $F=(1,0, \ldots, 0)$. Transposing both sides of (16) we obtain the equation $Z_{s} V^{\top}-V^{\top} J^{\top}=-F^{\top} E^{\top}$. Multiplying both sides of this equation with $W$ from the left, multiplying both sides of (20) with $V^{\top}$ from the right, and adding the equations gives the displacement equation for the matrix $W V^{\top}$ :

(21) $\Delta W V^{\top}-W V^{\top} J^{\top}=E F V^{\top}-W U^{\top} H^{\top}=\left(E \mid-W U^{\top}\right) \cdot\left(\begin{array}{c}F V^{\top} \\ H^{\top}\end{array}\right)=: G B$.

Written out explicitly, we have

$$
\begin{aligned}
& G=\left(\begin{array}{ccccc}
\epsilon_{1}^{D_{0}-1} & -\epsilon_{1}^{D_{1}-1} & -\epsilon_{1}^{D_{2}-1} & \ldots & -\epsilon_{1}^{D_{\ell}-1} \\
\epsilon_{2}^{D_{0}-1} & -\epsilon_{2}^{D_{1}-1} & -\epsilon_{2}^{D_{2}-1} & \cdots & -\epsilon_{2}^{D_{\ell}-1} \\
\vdots & \vdots & \vdots & \cdots & \vdots \\
\epsilon_{s}^{D_{0}-1} & -\epsilon_{s}^{D_{1}-1} & -\epsilon_{s}^{D_{2}-1} & \cdots & -\epsilon_{s}^{D_{\ell}-1}
\end{array}\right)
\end{aligned}
$$

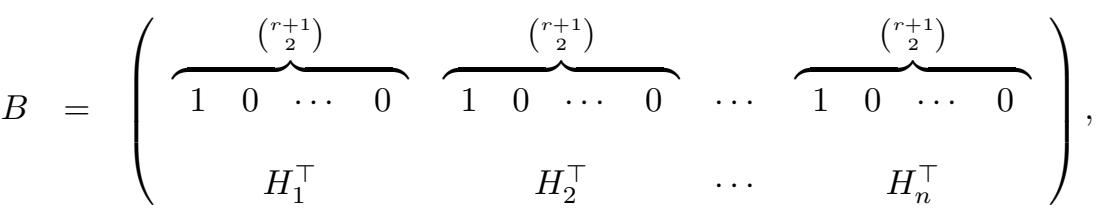

where $D_{0}:=0$, and $D_{i}:=d_{0}+\cdots+d_{i-1}$ for $i>0$, and $H_{i}$ as defined in (17).

The matrix $W V^{\top}$ has the right displacement structure, since $\Delta$ is diagonal and $J^{\top}$ is upper triangular. However, since we are interested in an element in the right kernel of $V$, we need to compute an element in the left kernel of $W V^{\top}$. This computation can be done using similar techniques as above by considering an appropriate displacement structure for the matrix $\left(W V^{\top} \mid I\right)$. However, certain complications arise in this case due to the fact that the displacement operator for this matrix which maintains a low displacement rank is not an isomorphism. Therefore, we defer the design of that algorithm to the appendix. Using that procedure, we obtain the following overall algorithm.

Algorithm 6.1. On input $\left(x_{1}, y_{1}\right), \ldots,\left(x_{n}, y_{n}\right) \in \mathbb{F}_{q}^{2}$ where none of the $x_{i}$ is zero, a positive integer $r$, and integers $d_{0}, d_{1}, \ldots, d_{\ell}$ such that $\sum_{i=0}^{\ell}\left(d_{i}+1\right)=: s>$ $\left(\begin{array}{c}r+1 \\ 2\end{array}\right) n$, this algorithm computes a polynomial $F(x, y)=\sum_{i=0}^{\ell} F_{i}(x) y^{i}$ such that $\operatorname{deg}\left(F_{i}\right)<d_{i}, F\left(x_{t}, y_{t}\right)=0$ for $t=1, \ldots, n$, and such that $F\left(x+x_{t}, y+y_{t}\right)$ does not have any monomial of degree less than $r$.

(1) Let $d:=\left\lceil\log _{q}(s+n+1)\right\rceil$ and construct the finite field $\mathbb{F}_{q^{d}}$.

(2) Choose $s$ pairwise distinct nonzero elements $\epsilon_{1}, \ldots, \epsilon_{s}$ in $\mathbb{F}_{q^{d}}$ such that $\epsilon_{i} \neq x_{j}$ for $i \neq j$.

(3) Run Algorithm A.1 on input

$$
D, J, G, B, \underbrace{(1,1, \ldots, 1)}_{\text {stimes }},
$$

where $D$ is the diagonal matrix with diagonal entries $1 / \epsilon_{1}, \ldots, 1 / \epsilon_{s}, J$ is the matrix defined in (15), $G$ is the matrix defined in (22), and $B$ is the matrix defined in (23). Let $w$ denote the output. 
(4) Compute $v:=w \cdot W$ where $W$ is the Vandermonde matrix with $(i, j)$-entry equal to $\epsilon_{i}^{j-1}$. Let $v=\left(v_{00}, v_{01}, \ldots, v_{0, d_{0}-1}, \ldots, v_{\ell, 0}, v_{\ell, 1}, \ldots, v_{\ell, d_{\ell}-1}\right) d e-$ note the output.

(5) Output $F(x, y)=\sum_{i=0}^{\ell} F_{i}(x) y^{i}$ where $F_{i}(x)=v_{i, 0}+v_{i, 1} x+\cdots+v_{i, d_{i}-1} x^{d_{i}-1}$.

THEOREM 6.2. Algorithm 6.1 correctly computes its output with $O\left(s^{2} \ell\right)$ operations over $\mathbb{F}_{q^{d}}$, or, equivalently, $O\left(s^{2} \ell \log _{q}(s)^{2}\right)$ operations over $\mathbb{F}_{q}$.

Proof. The finite field $\mathbb{F}_{q^{d}}$ can be constructed with a randomized algorithm with an expected number of $O\left(d^{3} \log (d) \log (d q)\right)$ operations over the field $\mathbb{F}_{q}[\mathbf{1 2}$, Th. 14.42]. This cost is negligible compared to the cost of the subsequent steps. As proved in the appendix, Step (3) requires $O\left(\ell s^{2}\right)$ operations over $\mathbb{F}_{q^{d}}$. Steps (4), (5), and (6) each require $O\left(s^{2}\right)$ operations over $\mathbb{F}_{q^{d}}$. Each $\mathbb{F}_{q^{d}}$-operation can be simulated with $O\left(d^{2}\right)$ operations over $\mathbb{F}_{q}$. The result follows.

\section{Parallel Algorithms}

The aim of this section is to show how the displacement method can be used to obtain parallel algorithms for computing a nontrivial element in the kernel of a structured matrix. The model of parallel computation that we use is a PRAM (Parallel Random Access Machine). A PRAM consists of several independent sequential processors, each with its own private memory, communicating with one another through a global memory. In one unit of time, each processor can read one global or local memory location, execute a single random access operation, and write into one global or local memory location. We assume in the following the each processor in the PRAM is capable of performing operations over $\mathbb{F}_{q}$ in a single step. The running time of a PRAM is the number of steps it performs to finish its computation.

We will use the following standard facts: one can multiply $m \times \alpha$ - with $\alpha \times k$ matrices on a PRAM in $O(\alpha k)$ time on $m$ processors. This is because each of the $m$ processors performs independently a vector-matrix multiplication of size $1 \times \alpha$ and $\alpha \times k$. Further, one can solve an upper triangular $m \times m$-system of equations in time $O(m)$ on $m$ processors. There are various algorithms for this problem. One is given in [29, Sect. 2.4.3]. As a result, it is easily seen that Algorithm 2.3 can customized to run in parallel, if steps (1) and (3) can be performed in parallel. More precisely, if these steps can be performed on $m$ processors in time $O(\alpha)$, then the whole algorithm can be customized to run in time $O(\alpha(m+n))$ on $m$ processors.

However, one cannot always perform steps (1) and (3) in parallel. Whether or not this is possible depends heavily on the displacement structure used. For instance, it is easy to see that the recovery algorithm given in Section 3 cannot be parallelized (at least in an obvious way). The reason for this is that for obtaining the $i$ th entry of the first row one needs to know the value of the $(i-1)$ st entry. To obtain the $n$th entry one thus needs to know the value of all the previous entries. It is therefore not possible to perform this step in time $O(\alpha)$ even if the number of processors is unbounded!

A closer look at this problem reveals the following: if $V$ has a displacement structure of the type $D V-V \Delta=G B$ with diagonal matrices $D$ and $\Delta$, then one can recover the first row and the first column of $V$ in parallel, if the nonzero entries of $D$ and $\Delta$ are pairwise distinct. The algorithm for this task is rather simple and is given below. 
The problem with this approach is that the matrices $V$ we are studying do not necessarily have low displacement structure with respect to a pair of diagonal matrices. This problem can be fixed in certain cases. For instance, suppose that $V \in \mathbb{F}_{q}^{m \times n}$ has low displacement rank with respect to $D$ and $Z_{n}$, where $D$ is diagonal and $Z_{n}^{q}$ is the upper shift matrix of format $n$ :

$$
D V-V Z_{n}=G_{1} B_{1} \text {. }
$$

Let $W$ be a Vandermonde matrix whose $(i, j)$-entry is $\epsilon_{i}^{j-1}$, where $\epsilon_{1}, \ldots, \epsilon_{n}$ are elements in $\mathbb{F}_{q}$ that are pairwise distinct and different from the diagonal entries of $D$. Then we have the following displacement structure for $W$

$$
\Delta W-W Z_{n}^{\top}=G_{2} B_{2}
$$

where $G_{2}=\left(\epsilon_{1}^{n}, \ldots, \epsilon_{n}^{n}\right)^{\top}$ and $B_{2}=(0,0, \ldots, 0,1)$. Transposing this equation and multiplying with $V$ from the left gives

$$
V Z_{n} W^{\top}-W^{\top} \Delta=\left(V B_{2}^{\top}\right) G_{2} .
$$

Multiplying (24) with $W$ from the right, and adding that to the previous equation gives

$$
D V W^{\top}-V W^{\top} \Delta=\left(G_{1} \mid V B_{2}^{\top}\right)\left(\begin{array}{c}
B_{1} W^{\top} \\
G_{2}
\end{array}\right)=: G B .
$$

This is now the correct displacement structure, but for the wrong matrix $V W^{\top}$. However, if $w$ is a nonzero vector in the kernel of $V W^{\top}$, then $v:=W^{\top} w$ is in the kernel of $V$. Moreover, since $W$ is invertible, $v$ is nonzero, hence is the desired solution.

The rest of this section deals with putting these ideas into effective use. We will first start by designing an algorithm that computes the first row and the first column of the matrix $V$ that has a displacement structure with respect to two diagonal matrices.

Algorithm 7.1. The input to the algorithm are two diagonal matrices $D$ and $\Delta$, and two additional matrices $G=\left(G_{i j}\right) \in \mathbb{F}_{q}^{m \times \alpha}$ and $B=\left(B_{i j}\right) \in \mathbb{F}_{q}^{\alpha \times n}$. We assume that note of the diagonal entries $x_{1}, \ldots, x_{n}$ is equal to any of the diagonal entries $z_{1}, \ldots, z_{m}$ of $\Delta$. The output of the algorithm are the first row $\left(r_{1}, \ldots, r_{m}\right)$ and the first column $\left(c_{1}, \ldots, c_{n}\right)^{\top}$ of the matrix $V$ given by the displacement structure $D V-V \Delta=G B$.

(1) Compute the first row $\left(\rho_{1}, \ldots, \rho_{m}\right)$ and the first column $\left.\left(\gamma_{1}, \ldots, \gamma_{n}\right)\right)^{\top}$ of $G B$.

(2) For $i=1, \ldots, n$ set in parallel $c_{i}:=\gamma_{i} /\left(x_{i}-z_{1}\right)$.

(3) For $i=1, \ldots, m$ set in parallel $r_{i}:=\rho_{i} /\left(x_{1}-z_{i}\right)$.

Proposition 7.2. The above algorithm correctly computes its output with $O(\alpha)$ operations on a PRAM with $\max \{m, n\}$ processors.

ProOF. Correctness of the algorithm is easily obtained the same way as that of Algorithm 3.1. As for the running time, note that Step (1) can be performed in time $O(\alpha)$ on $\max \{m, n\}$ processors. Steps (2) and (3) obviously need a constant number of steps per processor.

To come up with a memory-efficient procedure, we will customize Algorithm 2.5 rather than Algorithm 2.3. For this we need to have a displacement structure for 
$\left(\begin{array}{c}V \\ I_{n}\end{array}\right)$. This is given by the following:

$$
\left(\begin{array}{cc}
D & 0 \\
0 & \Delta
\end{array}\right)\left(\begin{array}{c}
V \\
I_{n}
\end{array}\right)-\left(\begin{array}{c}
V \\
I_{n}
\end{array}\right) \Delta=\left(\begin{array}{c}
G \\
0
\end{array}\right) B
$$

Algorithm 7.3. On input a diagonal matrix $D \in \mathbb{F}_{q}^{n \times n}$ with diagonal entries $x_{1}, \ldots, x_{n}$, a diagonal matrix $\Delta \in \mathbb{F}_{q}^{(n+1) \times(n+1)}$ with diagonal entries $z_{1}, \ldots, z_{n+1}$ such that the $x_{i}$ and the $z_{j}$ are pairwise distinct, and $z_{i} \neq x_{j}$ for $i \neq j$, a matrix $G \in \mathbb{F}_{q}^{n \times \alpha}$, and a matrix $B \in \mathbb{F}_{q}^{\alpha \times(n+1)}$, the algorithm computes a nonzero vector $v \in \mathbb{F}_{q}^{n}$ in the kernel of the matrix $V$ given by the displacement equation $D V-V \Delta=$ $G B$.

(1) Set $G_{0}:=\left(\begin{array}{c}G \\ 0\end{array}\right), B_{0}:=B$.

(2) For $i=0, \ldots, n-1 d o$ :

(a) Let $D_{i}$ be the diagonal matrix with diagonal entries $x_{i+1}, \ldots, x_{n}$, $z_{1}, \ldots, z_{i}$, and let $\Delta_{i}$ be the diagonal matrix with diagonal entries $z_{i+1}, \ldots, z_{n+1}$.

(a) Use Algorithm 7.1 to compute the first column $\left(c_{1}, c_{2}, \ldots, c_{2 n+1-i}\right)^{\top}$ of the matrix $V_{i}$ given by the displacement equation

$$
\left(\begin{array}{cc}
D_{i} & 0 \\
0 & \Delta
\end{array}\right) V_{i}-V_{i} \Delta_{i}=G_{i} B_{i} .
$$

(b) If $c_{1}=\cdots=c_{n-i}=0$, then output the vector

$v:=\left(c_{n-i+1}, \ldots, c_{n}, 1,0, \ldots, 0\right)$. and Stop. Otherwise, find the smallest $k$ such that $c_{k} \neq 0$, interchange $x_{1}$ and $x_{k}, c_{1}$ and $c_{k}$, and the first and the $k$ th rows of $G_{i}$.

(c) Use Algorithm 7.1 again to compute in parallel the first row $\left(r_{1}, \ldots, r_{n-i+1}\right)$ of the matrix $V_{i}$ given by $D_{i} V_{i}-V_{i} \Delta_{i}=G_{i} B_{i}$ (note that $V_{i}$ is not necessarily the same as in Step (2a) since $D_{i}$ and $G_{i}$ may not be the same).

(d) For $j=2, \ldots, 2 n-i+1$ replace row $j$ of $G_{i}$ by $-c_{j} / c_{1}$ times the first row plus the $j$-th row. Set $G_{i+1}$ as the matrix formed from $G_{i}$ by deleting the first row.

(e) For $j=2, \ldots, n-i+1$ replace the $j$-th column of $B_{i}$ by $-r_{j} / c_{1}$ times the first column plus the $j$-th column. Set $B_{i+1}$ as the matrix formed from $B_{i}$ by deleting the first column.

THEOREM 7.4. The above algorithm correctly computes its output and uses $O(\alpha n)$ operations on a PRAM with $n+1$ processors.

ProOF. This algorithm is a customized version of Algorithm 2.3 and its correctness follows from that of the latter. As for the running time, note that Step (2a) takes $O(\alpha)$ steps on a PRAM with $2 n+1-i$ processors by Proposition $7.2\left(G_{i}\right.$ has $2 n+1-i$ rows). However, a simple induction shows that the last $n+1-i$ rows of $G_{i}$ are zero. As a result, we only need $n-i$ processors for this step. Step (2c) can be performed in time $O(\alpha)$ on $n+1-i$ processors by Proposition 7.2. Steps $(2 \mathrm{~d})$ and $(2 \mathrm{e})$ can be performed in time $O(\alpha)$ on $n+1-i$ processors (again, the number of processors is not equal to the number of rows of $G_{i}$ since the last $n+1-i$ rows of $G_{i}$ are zero). At each round of the algorithm the processors can be reused, so the total number of processors equals to the maximum number at each round, i.e., 
it equals $n+1$. The running time is at most $O(n \alpha)$ since step (2) is performed at most $n$ times.

The above algorithm can now be used as a major building block for solving the 2-dimensional interpolation problem in parallel.

Algorithm 7.5. On input $\left(x_{1}, y_{1}\right), \ldots,\left(x_{n}, y_{n}\right) \in \mathbb{F}_{q}^{2}$ and integers $d_{0}, d_{1}, \ldots, d_{\ell}$ such that none of the $x_{i}$ is zero and such that $\sum_{i=0}^{\ell} d_{i}=n+1$, this algorithm computes a polynomial $F(x, y)=\sum_{i=0}^{\ell} F_{i}(x) y^{i}$ such that $\operatorname{deg}\left(F_{i}\right)<d_{i}$ for $i=$ $0, \ldots, \ell$ and $F\left(x_{t}, y_{t}\right)=0$ for $t=1, \ldots, n$.

(1) Let $d:=\left\lceil\log _{q}(2 n+1)\right\rceil$ and construct the field $\mathbb{F}_{q^{d}}$. Find $n+1$ elements $\epsilon_{1}, \ldots, \epsilon_{n+1}$ in $\mathbb{F}_{q^{d}}$ that are pairwise distinct and such that $\epsilon_{i} \neq 1 / x_{j}$ for $i \neq j$.

(2) Set $G:=\left(G_{1} \mid v_{1}\right)$ where $G_{1}$ is the matrix given in (11) and $v_{1}$ is the last column of the matrix $V$ given in (10).

(3) Compute $\tilde{B}:=B_{1} W^{\top}$ where $B$ is the matrix given in (12) and $W$ is the $(n+1) \times(n+1)$ Vandermonde matrix having $(i, j)$-entry $\epsilon_{i}^{j-1}$. Set $B:=\left(\begin{array}{c}\tilde{B} \\ G_{2}\end{array}\right)$ where $G_{2}$ is the vector $\left(\epsilon_{1}^{n+1}, \ldots, \epsilon_{n+1}^{n+1}\right)$.

(4) Run Algorithm 7.3 on input $D, \Delta, G, B$, where $D$ is a diagonal matrix with diagonal entries $1 / x_{1}, \ldots, 1 / x_{n}, \Delta$ is the diagonal matrix with diagonal entries $\epsilon_{1}, \ldots, \epsilon_{n+1}$, and $G$ and $B$ are the matrices computed in the previous two steps. Let $w$ denote the output.

(5) Compute in parallel the vector $v:=W^{\top} w$. Let $\left(v_{00}, v_{01}, \ldots, v_{0, d_{0}-1}, \ldots, v_{\ell, 0}, v_{\ell, 1}, \ldots, v_{\ell, d_{\ell}-1}\right)$ denote its components.

(6) Output $F(x, y)=\sum_{i=0}^{\ell} F_{i}(x) y^{i}$ where $F_{i}(x)=v_{i, 0}+v_{i, 1} x+\cdots+v_{i, d_{i}-1} x^{d_{i}-1}$.

THEOREM 7.6. The above algorithm correctly computes its output with $O\left(\ln \log _{q}(n)^{2}\right)$ operations on a PRAM with $n+1$ processors.

Proof. The matrix $V W^{\top}$ satisfies the displacement equation 26 with the matrices $G$ and $B$ computed in steps (2) and (3). Step (4) then produces a nontrivial element in the kernel of $V W^{\top}$. This shows that the vector $v$ computed in Step (5) is indeed a nontrivial element in the right kernel of $V$, and proves correctness of the algorithm.

The finite field $\mathbb{F}_{q^{d}}$ can be constructed probabilistically in time proportional to $d^{3} \log (q d) \log (d)$ on one processor [12, Th. 14.42]. Arithmetic operations in $\mathbb{F}_{q^{d}}$ can be simulated using $d^{2}$ operations over $\mathbb{F}_{q}$ on each processor. In the following we thus assume that the processors on the PRAM are capable of executing one arithmetic operation in $\mathbb{F}_{q^{d}}$ in $O\left(d^{2}\right)$ steps. In Step (2) $G_{1}$ can be calculated with $O(\alpha \log (n)) \mathbb{F}_{q^{d}}$ operations on $n$ processors (each of which computes the rows of $G_{1}$ independently). Likewise, $v_{1}$ can be computed with $O(\log (n))$ operations on $n$ processors. Hence, computation of $G$ requires $O(\ell \log (n))$ operations over $\mathbb{F}_{q^{d}}$. Given the special structure of the matrix $B_{1}, \tilde{B}$ can be computed with $O(n) \mathbb{F}_{q^{d}}$ operations on $n+1$ processors. $G_{2}$ can be computed with $O(\log (n))$ operations on $n+1$ processors. Hence, steps $(2)$ and $(3)$ require $O(\ell \log (n)+n)$ operations on $n+1$ processors. Step $(4)$ needs $O(\ell n)$ operations on $(n+1)$ processors by Theorem 7.4 , and Step (5) needs $O(n)$ operations on $n+1$ processors. Hence, the total number

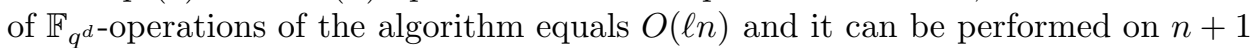
processors. 


\section{References}

[1] S. Ar, R. Lipton, R. Rubinfeld, and M. Sudan. Reconstructing algebraic functions from mixed data. In Proceedings of the 33rd IEEE Symposium on Foundations of Computer Science, pages 503-512, 1992.

[2] D. Augot and L. Pecquet. An alternative to factorisation: a speedup for Sudan's algorithm and its generalization to algebraic-geometric codes. Preprint, 1998.

[3] E.R. Berlekamp. Algebraic Coding Theory. McGraw-Hill, New York, 1968.

[4] E. R. Berlekamp and L. Welch. Error correction of algebraic block codes. US Patent Number 4,633,470, 1986.

[5] E.R. Berlekamp. Bounded distance +1 soft decision Reed-Solomon decoding. IEEE Trans. Inform. Theory, 42:704-720, 1996.

[6] R. Bitmead and B. Anderson. Asymptotically fast solution of Toeplitz and related systems of linear equations. Linear Algebra and its Applications, 34:103-116, 1980.

[7] J.M.Delosme. Algorithms and Implementations for Liner Least Squares Estimation. Ph.D.Thesis, Stanford University, 1982

[8] H.Dym. Structured matrices, reproducing kernels and interpolation, Structured Matrices in Mathematics, Computer Science, and Engineering, AMS series CONTEMPORARY MATHEMATICS, vol. 280, pp. 3-30, AMS Publications, 2001.

[9] B.Friedlander, M.Morf, T.Kailath and L.Ljung, New inversion formulas for matrices classified in terms of their distance from Toeplitz matrices, Linear Algebra and its Applications, 27 (1979) 31-60.

[10] G. Golub and C. van Loan Matrix computations, third edition, The Johns Hopkins University Press Ltd., London, 1996.

Computing roots of polynomials over function fields of curves. In David Joyner, editor, Coding Theory and Cryptography: from Enigma and Geheimschreiber to Quantum Theory, pages 214-228. Springer Verlag, 1999.

[11] S. Gao and M.A. Shokrollahi. Computing roots of polynomials over function fields of curves. In David Joyner, editor, Coding Theory and Cryptography: from Enigma and Geheimschreiber to Quantum Theory, pages 214-228. Springer Verlag, 1999.

[12] J. von zur Gathen and J. Gerhard. Modern Computer Algebra. Cambridge Univ. Press, Cambridge, 1999.

[13] I. Gohberg and V. Olshevsky. Fast algorithm for matrix Nehari problem, roceedings of MTNS93, Systems and Networks: Mathematical Theory and Applications, v.2, Invited and Contributed Papers,edited by U. Helmke, R. Mennicken and J. Sauers, Academy Verlag,1994, p. 687-690.

[14] I. Gohberg and V. Olshevsky. Fast state-space algorithms for matrix Nehari and NehariTakagi interpolation problems. Integral Equations and Operator Theory, 20:44-83, 1994.

[15] V. Guruswami and M. Sudan. Improved decoding of Reed-Solomon and algebraic-geometric codes. In Proceedings of the 39th IEEE Symposium on Foundations of Computer Science, pages 28-37, 1998.

[16] G. Heinig Ein Prinzip zur Invertierung einiger Klassen linearer Operatoren, Proceedings of the 7th Conference on Methods in Mathematical Physics (7th TMP), TH Karl-Marx-Stadt 1979, vol.2, pp.45-50.

[17] G. Heinig and K. Rost. Algebraic Methods for Toeplitz-like matrices and operators, volume 13 of Operator Theory. Birkhäuser, Boston, 1984.

[18] T. Høholdt and R. Refslund Nielsen. Decoding Hermitian codes with Sudan's algorithm. Preprint, Denmark Technical University, 1999.

[19] T. Høholdt and R. Pellikaan. On the decoding of algebraic-geometric codes. IEEE Trans. Inform. Theory, 41:1589-1614, 1995.

[20] J. Justesen, K.J. Larsen, H.E. Jensen, and T. Høholdt. Fast decoding of codes from algebraic plane curves. IEEE Trans. Inform. Theory, 38:111-119, 1992.

[21] T. Kailath, Some new algorithms for recursive estimation in constant linear systems, IEEE transactions on Information Theory, IT-19, Nov. 1973, 750-760.

[22] T. Kailath and J. Chun, Generalized Displacement Structure for Block-Toeplitz, ToeplitzBlock, and Toeplitz-Derived Matrices, SIAM J. Matrix Anal. Appl., vol. 15, no. 1, pp. 114128, January 1994. 
[23] T.Kailath, S.Kung and M.Morf, Displacement ranks of matrices and linear equations, $J$. Math. Anal. and Appl., 68 (1979) 395-407.

[24] T. Kailath and V. Olshevsky. Fast Gaussian Elimination with Partial Pivoting for Matrices with Displacement Structure Mathematics of Computation, 64 No. 212 (1995), 1557-1576.

[25] T. Kailath and V. Olshevsky. Displacement structure approach to discrete trigonometric transform based preconditioners of G. Strang and T. Chan types. Calcolo, 33:191-208., 1996.

[26] T. Kailath and V. Olshevsky. Unitary Hessenberg matrices and the generalized Parker-ForneyTraub and Bjorck-Pereyra algorithms for Szego-Vandermonde matrices. To appear. Can be obtained from http://www.cs.gsu.edu/ ${ }^{\sim}$ matvro, 1997.

[27] T. Kailath and A.H. Sayed. Displacement structure: Theory and applications. SIAM Review, 37:297-386, 1995.

[28] R. Kötter. Fast generalized minimum-distance decoding of algebraic-geometry and ReedSolomon codes. IEEE Trans. Inform. Theory, 42:721-737, 1996.

[29] F.T. Leighton. Introduction to Parallel Algorithms and Architectures : Arrays, Trees, Hypercubes. Academic Press/Morgan Kaufmann, 1992.

[30] H.Lev Ari. Nonstationary Lattice-Filter Modeling. Ph.D.Thesis, Stanford University, 1983

[31] H. Lev-Ari, Displacement Structure: Two Related Perspectives, in Communications, Computing, Control and Signal Processing: A Tribute to Thomas Kailath, A. Paulraj, V. Roychowdhury and C. Schaper, eds., pp. 233-241, Kluwer Academic Publishers, Norwell, MA, 1997.

[32] F.J. MacWilliams and N.J.A. Sloane. The Theory of Error-Correcting Codes. North-Holland, 1988.

[33] M. Morf. Fast algorithms for multivariable systems. PhD thesis, Stanford University, 1974.

[34] M. Morf. Doubling algorithms for Toeplitz and related equations. In Prceedings of IEEE Conference on Acoustics, Speech, and Signal Processing, Denver, pages 954-959, 1980.

[35] V. Olshevsky (editor). Structured Matrices in Mathematics, Computer Science, and Engineering, AMS series CONTEMPORARY MATHEMATICS, vols. 280, 281, AMS Publications, 2001.

[36] V. Olshevsky Pivoting for structured matrices with applications. http://www.cs.gsu.edu/ ${ }^{\text {matvro, } 1997 .}$

[37] V. Olshevsky and V. Pan. A superfast state-space algorithm for tangential Nevanlinna-Pick interpolation problem. In Proceedings of the 39th IEEE Symposium on Foundations of Computer Science, pages 192-201, 1998.

[38] V. Olshevsky and M.A. Shokrollahi. A displacement structure approach to list decoding of algebraic-geometric codes. In Proceedings of the 31st Annual ACM Symposium on Theory of Computing, pages 235-244, 1999.

[39] R. Roth and G. Ruckenstein. Efficient decoding of reed-solomon codes beyond half the minimum distance. In Prceedings of 1998 International Symposium on Information Theory, pages 56-56, 1998.

[40] L.A. Sakhnovich, On similarity of operators (in Russian), Sibirskij Mat.J. 8 , 4 (1972), 8686883.

[41] L.A. Sakhnovich, On the integral equation with kernel depending on the difference of the arguments (in Russian), Matem. Issledovanya, Kishinev, 8, 2 (1973), 138-146.

[42] L.A. Sakhnovich, The factorization of the operator-valued transfer functions, Soviet Math. Dokl., 17 (1976), 203-207.

[43] L.A. Sakhnovich, Factorization problems and operator identities, Russian Mathematical Surveys, 41, 1 (1986), $1-64$

[44] M.A. Shokrollahi and H. Wasserman. List decoding of algebraic-geometric codes. IEEE Trans. Inform. Theory, 45:432-437, 1999.

[45] M. Sudan. Decoding of Reed-Solomon codes beyond the error-correction bound. J. Compl., 13:180-193, 1997.

[46] L.R. Welch and E.R. Berlekamp. Error correction for algebraic block codes. U.S. Patent 4,633,470, issued Dec. 30, 1986. 


\section{Appendix A}

In this appendix we shall clarify how to implement Step 3 of Algorithm 6.1, i.e., how to find a vector in the right kernel of a matrix $R:=V W^{\top}$ satisfying

$$
J R-R \Delta=G B
$$

(cf. (21)). By Lemma 2.4 we could use the extended displacement equation

$$
\left(\begin{array}{cc}
J & 0 \\
0 & \Delta
\end{array}\right)\left(\begin{array}{c}
R \\
I
\end{array}\right)-\left(\begin{array}{c}
R \\
I
\end{array}\right) \Delta=\left(\begin{array}{c}
G \\
0
\end{array}\right) B
$$

to try to run the algorithm 2.5 to compute a vector in the right kernel of $R:=V W^{\top}$. Indeed, the latter algorithm is based on Lemma 2.1 which is directly applicable in our situation because $\left(\begin{array}{cc}J & 0 \\ 0 & \Delta\end{array}\right)$ is lower triangular, and $\Delta$ is upper triangular (even diagonal).

However, there are several differences between (28) and (5) that do not allow one to apply the Algorithm 2.5 directly.

(1) A closer look at (28) reveals that the mapping $\nabla: \mathbb{F}^{(m+s) \times s} \longrightarrow \mathbb{F}^{(m+s) \times \alpha} \times$ $\mathbb{F}^{\alpha \times s}$ defined by $\nabla S=\left(\begin{array}{cc}J & 0 \\ 0 & \Delta\end{array}\right) S-S \Delta$ is not an isomorphism, so the the generator $\left\{\left(\begin{array}{c}G \\ 0\end{array}\right), B\right\}$ no longer contains the full information about $\left(\begin{array}{c}R \\ I\end{array}\right)$ in (28). Thus, we need to introduce a new definition for a generator,

$$
\left\{\left(\begin{array}{c}
G \\
0
\end{array}\right), B, D\right\}
$$

including in it one more matrix $D$ to completely describe our structured $\operatorname{matrix}\left(\begin{array}{c}R \\ I\end{array}\right)$.

(2) Lemma 2.4 shows how to compute a vector in the kernel of $R$ via computing the Schur complements of $\left(\begin{array}{c}R \\ I\end{array}\right)$. Further, Lemma 2.1 allows us to speed-up the procedure by manipulation on two generator matrices $\left\{\left(\begin{array}{c}G \\ 0\end{array}\right), B\right\}$. In our situation (having a new extended generator $(29)$ ) we need to specify a recursion for the third matrix in (29) as well.

(3) One of the ingredients of the algorithm 2.5 is recovering the first row and the first column of a structured matrix from its generator. We need to clarify this procedure for our new extended generator in (29).

(4) In what follows we provide formulas resolving the above three difficulties, and specify a modified version of the fast algorithm 2.5 for our matrix $S:=\left(\begin{array}{c}R \\ I\end{array}\right)$. However, the original algorithm 2.5 employes partial row pivoting to run to the completion. ${ }^{1}$ Row pivoting was possible due to the

\footnotetext{
${ }^{1}$ Specifically, at each step the algorithm 2.5 uses row interchanges to bring a nonzero element in the $(1,1)$ position of $S$ (moreover, the absence of appropriate non-zero elements was the stopping criterion).
} 
diagonal form of the matrix $D$ in (5). In contrast, the new matrix $J$ in (28) is no longer diagonal, so we have to find a different pivoting strategy. We next resolve the above four difficulties. Let $S$ satisfy

$$
A S-S \Delta=G B \text {, }
$$

where we set $A:=\left(\begin{array}{cc}J & 0 \\ 0 & \Delta\end{array}\right) \in \mathbb{F}^{(n+s) \times(n+s)}$ and $\Delta \in \mathbb{F}^{s \times s}$ is diagonal.

(1) A general theory of displacement equations with kernels can be found in $[\mathbf{3 6}, \mathbf{2 6}]$, and this more delicate case appears in studying boundary rational interpolation problems and in the design of preconditioners [25]. As we shall see below, we can recover from $\{G, B\}$ on the right-hand side of (30) all the enries of the $(n+s) \times s$ matrix $S$ but the $s$ diagonal elements $\{S(n+1,1), S(n+2,2), \ldots, S(n+s, s)\}$. Therefore the triple

$$
\{G, B, D\} \quad \text { with } \quad D:=\{S(n+1,1), S(n+2,2), \ldots, S(n+s, s)\}
$$

contains the full information on $S$, so we call it a generator.

It should be mentioned that the idea of generator is in compressing the information on $n^{2}$ entries of $S$ into just $O(n)$ entries of its generator. The newly defined generator in (31) involves just $s$ more parameters (i.e., the entries of the $1 \times s$ row $D$ ) and therefore is still a very efficient representation.

(2) Each step of the algorithm 2.5 consists of two parts: (1) recovering the first row and column of $S$ from its generator; (2) computing a generator $\left\{G_{2}, B_{2}, D_{2}\right\}$ for the Schur complement $S_{2}$ of $S$. We shall discuss part (1) in the next item, and here we address part (2). A closer look at lemma 2.1 reveals that it applies in our situation and the formulas for computing $\left\{G_{2}, B_{2}\right\}$ remain valid. It remains to show how to compute the row $D_{2}=$ $\left\{d_{1}^{(2)}, d_{2}^{(2)}, \ldots, d_{s}^{(2)}\right\}$ capturing the $\{(n+1,1),(n+2,2), \ldots,(n+s-1, s-1)\}$ elements of $S_{2}$. Since $S_{2}=S_{22}-S_{21} S_{11}^{-1} S_{12}$ is the Schur complement of $S=\left(\begin{array}{ll}S_{11} & S_{12} \\ S_{21} & S_{22}\end{array}\right)$, we have the following formulas

$$
d_{k}^{(2)}=S_{n+k, k}-S_{n+k, 1} S_{1,1}^{-1} S_{1, k}
$$

where all the involved entries $S_{i j}$ of $S$ are available: $\left\{S_{n+k, 1}, S_{1,1}, S_{1, k}\right\}$ appear either in the first row or in the first column (we shall show next how to recover them from $(31)$ ), and $S_{n+k, k}$ is an entry of the matrix $D$ in $(31)$.

(3) Now we are able to provide formulas to recover the first row and column of $S$ in (30) from its generator in (31).

The elements of the top row can be obtained by the formula

$$
S(1, k)=\frac{G(1,:) B(:, k)}{A(1,1)-\Delta(1, k)},
$$

where we follow the MATLAB notation and denote by $G(1,:)$ the first row of $G$ and by $B(:, k)$ the $k$-th column of $B$. This formula works only if $A_{1,1} \neq \Delta_{1, k}$. If $A_{1,1}=\Delta_{1, k}$ (this may happen only for $k=1$ ) then $S_{1,1}$ cannot be recovered from $\{G, B\}$ and is therefore stored in the third matrix $D$ of $(31)$. 
Since $A$ is a bi-diagonal matrix, the elements of the first column of $S$ can be recovered as follows:

$$
S(1,1)=\frac{G(1,:) B(:, 1)}{A(1,1)-\Delta(1,1)}, \quad S(k, 1)=\frac{[G(k,:)-A(k, k-1) G(k-1,:)] B(:, 1)}{A(k, k)-\Delta(1,1)}
$$

(4) The above items describe several modifications to the algorithm 2.5. The modified algorithm terminates successfully after $k$ steps when the first $m-k$ entries of the $(m-k+s) \times s$ Schur complement are zero (cf. Lemma 2.4)). However, we need to implement an appropriate pivoting strategy to ensure that at each step of the algorithm, the element $S(1,1)$ of the Schur complement is non-zero. Pivoting is the standard method to bring a non-zero element in the $(1,1)$ position, however, arbitrary pivoting can destroy the structure of $S$ and thus block further efficient steps of the algorithm. Indeed, lemma 2.1 requires that the matrix $A$ is lower triangular and the matrix $\Delta$ in (30) is upper triangular, which can be destroyed by row/column interchanges. In our case the matrix $\Delta$ is diagonal, so any $\left(P^{\top} \Delta P\right)$ is diagonal as well. Therefore the permuted $(S P)$ satisfies

$$
A(S P)-(S P)\left(P^{\top} \Delta P\right)=G(B P) .
$$

and thus pivoting can be easily implemented in terms of operations on the generator of $S$. However, the above column pivoting does not remove all difficulties, and it may happen that the entire first row of $S$ is zero although there are still non-zero entries in the first column of $S$. In this case one may proceed as follows. Since the top row of $S$ is zero, we are free to make any changes in the first column of $A$ in (30). Let us zero all the entries of $A(:, 1)$ but $A(1,1)$. Then $A$ becomes a block-diagonal matrix. Denote by $Q$ a permutation that cyclically shifts tows 1 through $s$ of $A$. Then (30) implies

$$
\left(Q A Q^{\top}\right)(Q S)-(Q S) \Delta=(Q G) B
$$

and $\left(Q A Q^{\top}\right)$ is still lower triangular.

Thus applying column pivoting we bring the non-zero entries in the first row to the $(1,1)$ position, and applying row pivoting we move all zeros to the bottom. Note that we do not need to re-compute the entries $(n+1,1), \ldots,(n+s-1, s-1)$ of $Q S$, since they are the same as the corresponding entries of $S$. Further, since the zero rows remain zero in the Schur complement of $S$, they are not considered later in the process of the algorithm, this modified version has a running time of $O(\alpha n(n+s))$, where $\alpha$ is the displacement rank of $S$.

Altogether, we obtain the following algorithm:

Algorithm A.1. On input integers $n, s, n<s$, a diagonal matrix $\Delta \in \mathbb{F}_{q}^{s \times s}$ with diagonal entries $x_{1}, \ldots, x_{s}$, a lower triangular Jordan matrix $J \in \mathbb{F}_{q}^{n \times n}$, a matrix $G \in \mathbb{F}_{q}^{(n+s) \times \alpha}$, a matrix $B \in \mathbb{F}_{q}^{\alpha \times s}$, and a vector $d:=\left(d_{1}, \ldots, d_{s}\right)$, the algorithm computes a nonzero vector $v \in \mathbb{F}_{q}^{s}$ such that the matrix $S$ given by the displacement equation

$$
A S-S \Delta=G B, \quad A:=\left(\begin{array}{cc}
J & 0 \\
0 & \Delta
\end{array}\right)
$$


and having entries $(n+1,1), \ldots,(n+s, s)$ equal to $d_{1}, \ldots, d_{s}$.

(1) Set $G_{1}:=G, B_{1}:=B, d^{1}:=d, \Delta_{1}:=\Delta$, and $A_{1}:=A, S_{1}:=S$.

(2) For $i=1, \ldots, n$ do

(a) Compute the first row $\left(r_{1}, \ldots, r_{s-i+1}\right)$ and the first column $\left(c_{1}, \ldots, c_{n+s-i+1}\right)^{\top}$ of $S_{i}$ using (33) and (34).

(b) If $c_{1}=\cdots=c_{n-i+1}=0$, then output $\left(c_{n-i+2}, \ldots, c_{n+s-i+1}\right)^{\top}$ and STOP.

(c) If the first row is nonzero, find smallest $k$ such that $r_{k} \neq 0$, interchange $r_{1}$ and $r_{k}$, columns 1 and $k$ of $B_{i}$, and diagonal entries 1 and $k$ of $\Delta_{i}$.

(d) While the first row of $S_{i}$ is zero, cyclically shift $c_{1}, \ldots, c_{n-i+1}$, rows $1, \ldots, n-i+1$ of $G_{i}$, delete position $(2,1)$ of $A_{i}$ and interchange diagonal entries 1 and $n-i+1$ of $A_{i}$. At each step, compute the first row of the new $S_{i}$. (This while loop stops since the first column of $S_{i}$ is nonzero.)

(e) For $j=2, \ldots, n+s-i+1$ replace row $j$ of $G_{i}$ by $-c_{j} / c_{1}$ times the first row plus the $j$-th row. Set $G_{i+1}$ as the matrix formed from $G_{i}$ by deleting the first row.

(f) For $j=2, \ldots, s-i+1$ replace the $j$-th column of $B_{i}$ by $-r_{j} / c_{1}$ times the first column plus the $j$-th column. Set $B_{i+1}$ as the matrix formed from $B_{i}$ by deleting the first column.

(g) Compute the new values $d^{i+1}=\left(d_{1}^{\prime}, \ldots, d_{s}^{\prime}\right)$ from (32).

(h) Set $\Delta_{i+1}$ as the digonal matrix with entries $x_{i+1}, \ldots, x_{s}$. Set $A_{i+1}$ as the matrix obtained from $A$ by deleting the first row and the first column. Let $S_{i+1}$ denote the matrix satisfying the displacement equation $A_{i+1} S_{i+1}-S_{i+1} \Delta_{i+1}=G_{i+1} B_{i+1}$ and having entries $(n-i+$ $2,1), \ldots,(n+s-i+1, s)$ equal to $d_{1}^{\prime}, \ldots, d_{s}^{\prime}$.

Department of Mathematics, University of Connecticut, Storrs, CT 06269

E-mail address: olshevsky@math.uconn.edu

Digital Fountain, 39141 Civic Center Drive, Fremont, CA 94538

E-mail address: amin@digitalfountain.com 